NBER WORKING PAPER SERIES

\title{
INACCURATE STATISTICAL DISCRIMINATION: AN IDENTIFICATION PROBLEM
}

\author{
J. Aislinn Bohren \\ Kareem Haggag \\ Alex Imas \\ Devin G. Pope \\ Working Paper 25935 \\ http://www.nber.org/papers/w25935 \\ NATIONAL BUREAU OF ECONOMIC RESEARCH \\ 1050 Massachusetts Avenue \\ Cambridge, MA 02138 \\ June 2019
}

We thank Alex Frankel, Emir Kamenica, Emily Nix, and seminar participants at Harvard Business School, Harvard Kennedy School, the SaMMF Discrimination in Labor Markets Workshop, Stanford University, UCLA, University of Chicago, University of Melbourne, University of Pennsylvania, University of Southern California, University of Sydney, University of Virginia and the Virtual Market Design Seminar for helpful comments and suggestions. Cuimin $\mathrm{Ba}$ and Jihong Song provided excellent research assistance.The views expressed herein are those of the authors and do not necessarily reflect the views of the National Bureau of Economic Research.

NBER working papers are circulated for discussion and comment purposes. They have not been peerreviewed or been subject to the review by the NBER Board of Directors that accompanies official NBER publications.

(C) 2019 by J. Aislinn Bohren, Kareem Haggag, Alex Imas, and Devin G. Pope. All rights reserved. Short sections of text, not to exceed two paragraphs, may be quoted without explicit permission provided that full credit, including $\odot$ notice, is given to the source. 
Inaccurate Statistical Discrimination: An Identification Problem

J. Aislinn Bohren, Kareem Haggag, Alex Imas, and Devin G. Pope

NBER Working Paper No. 25935

June 2019, Revised July 2020

JEL No. D90,J71

\begin{abstract}
$\underline{\text { ABSTRACT }}$
Discrimination has been widely studied in the social sciences. Economists often categorize the source of discrimination as either taste-based or statistical-a valuable distinction for policy design and welfare analysis. In this paper, we highlight that in many situations economic agents may have inaccurate beliefs, and demonstrate that the possibility of inaccurate statistical discrimination generates an identification problem for attempts to isolate the source of differential treatment. We introduce isodiscrimination curves - which represent the set of preferences and beliefs that generate the same level of discrimination-to formally outline the identification problem: when not accounted for, inaccurate statistical discrimination can be mistaken for tastebased discrimination, accurate statistical discrimination, or their combination. A review of the empirical discrimination literature in economics, spanning 1990-2018, reveals the scope of this issue. While most papers discuss and attempt to distinguish between taste and statistical discrimination, a small minority-fewer than 7\%-consider inaccurate beliefs in the analysis. An experiment illustrates a methodology for differentiating between the three sources of discrimination, demonstrating the pitfalls of the identification problem while presenting a portable solution.
\end{abstract}

J. Aislinn Bohren

Carnegie Mellon University and

University of Pennsylvania

4765 Forbes Avenue

Pittsburgh, PA 15213

abohren@gmail.com

Kareem Haggag

Social and Decision Sciences

Carnegie Mellon University

Porter Hall 208-H

5000 Forbes Avenue

Pittsburgh, PA 15213

kareem.haggag@cmu.edu

\author{
Alex Imas \\ Social and Decision Sciences \\ Carnegie Mellon University \\ Porter Hall 319C \\ 500 Forbes Ave. \\ Pittsburgh, PA 15213 \\ aimas@andrew.cmu.edu \\ Devin G. Pope \\ Booth School of Business \\ University of Chicago \\ 5807 South Woodlawn Avenue \\ Chicago, IL 60637 \\ and NBER \\ devin.pope@chicagobooth.edu
}

A Data Appendix is available at http://www.nber.org/data-appendix/w25935 


\section{Introduction}

Economists define discrimination as differential treatment of otherwise identical individuals from different social groups (i.e. race, gender, age, etc.). Discrimination has been shown to be prevalent in labor markets, housing markets, credit markets, and online consumer markets among others. A variety of empirical techniques have been used to document discrimination, including correspondence studies, own-group bias, correspondence studies and observational data analysis (for recent reviews of the discrimination literature, see Bertrand and Duflo (2017); Charles and Guryan (2011)).

In addition to causally establishing the existence of discrimination, economists typically categorize discrimination as one of two types. The first type, taste-based discrimination (Becker, 1957), posits that an individual or firm has animus towards members of a particular group, and therefore may choose to discriminate against them because he receives disutility from providing services to or interacting with members of the group. The second type, statistical discrimination, suggests that discrimination may occur against members of a particular group because productivity is unobserved and the group is correctly perceived to have a lower average productivity due to exogenous differences (Phelps, 1972) or as part of a self-fulfilling equilibrium (Arrow, 1973); alternatively, the group's productivity may be perceived to have a different variance (Aigner and Cain, 1977) or the precision of auxiliary information about individual productivity may be perceived to differ.

Distinguishing between these forms of discrimination is important for several reasons. First, designing an effective policy intervention to reduce discrimination crucially depends on the source of discrimination. Second, welfare and efficiency analyses differ as a function of the source. For example, statistical discrimination is sometimes referred to as "efficient discrimination," in that it is the optimal response to a signal-extraction problem. Importantly, statistical discrimination is typically assumed to be driven by rational expectations, or correct beliefs, about the group distributions of the relevant outcome. Finally, the extent to which competitive markets will eliminate discrimination depends on its source (see Fang and Moro (2011) for review).

In this paper, we argue that in many situations, an individual's beliefs about the productivity of different social groups may not be correct. We refer to discrimination that stems from incorrect beliefs as inaccurate statistical discrimination. Just as it is important to distinguish between taste-based and accurate statistical discrimination for policy design and welfare analysis, we show that it is also critically important to separate inaccurate from accurate statistical discrimination. For example, if discrimination stems from inaccurate be- 
liefs, an effective policy response could be to provide individuals with information about the correct distributions. ${ }^{1}$ Moreover, as we formally show in Section 3, allowing for beliefs to be inaccurate generates an identification problem for common methods used to isolate the source of discrimination.

Two broad sources may lead to inaccurate beliefs. First, research in psychology has shown that biases and heuristics may generate beliefs that are systematically incorrect, leading to inaccurate stereotypes about certain groups (Schneider, Hastorf, and Ellsworth, 1979; Judd and Park, 1993; Hilton and Hippel, 1996); see Fiske (1998) for review. Bordalo, Coffman, Gennaioli, and Shleifer (2016) provide a model for inaccurate stereotype formation based on the representativeness heuristic. Biased beliefs can also arise in a dynamic learning setting when individuals either have incorrect models of how others evaluate workers (Bohren, Imas, and Rosenberg, 2019) or have different updating rules that depend on group identity (Albrecht, Von Essen, Parys, and Szech, 2013). Second, inaccurate beliefs may simply be due to a lack of information. A completely rational actor may lack the relevant information necessary to form correct beliefs. For example, an employer may have an unbiased prior belief about the average productivity of individuals from two different social groups, but be unaware that there is positive selection into the job application process for members of one group. This employer will be less likely to hire members of a particular group because she has an inaccurate belief about productivity differences due to a lack of information about the selection process.

Regardless of their source, inaccurate beliefs have been shown to exist in a variety of important domains, including the value of human capital formation (Jensen, 2010), the prevalence of affirmative action (Kravitz and Platania, 1993), and the extent of wealth inequality in the US (Norton and Ariely, 2011). Learning will mitigate inaccurate beliefs in some settings. But in other situations, there will be little or no feedback on the decisions being made, leading to learning traps in which inaccurate beliefs persist. Inaccurate beliefs may persist in the long run even when employers are perfectly Bayesian if, for example, they face a trade off between learning about the productivity of groups through hiring or maximizing cost-effectiveness (Lepage, 2020). Further, as shown theoretically by GagnonBartsch, Rabin, and Schwartzstein (2018), learning may not correct beliefs if information is filtered through an incorrect model of the world. Moreover, besides affecting the level of discrimination of the person holding them, inaccurate beliefs can have negative spillovers on

\footnotetext{
${ }^{1}$ See for example, Jensen (2010) in the case of inaccurate beliefs about the returns to education, or Bursztyn, González, and Yanagizawa-Drott (2018) in the case of inaccurate beliefs about the beliefs of others, i.e. pluralistic ignorance
} 
the discrimination of other evaluators if people learn from both signals and the actions of others (Hübert and Little, 2020).

To examine the extent to which inaccurate beliefs have been considered as a source of discrimination, we conducted an in-depth review of the economics literature on discrimination, spanning ten journals and nearly three decades (1990-2018) of papers. Section 2 presents our findings. The vast majority of papers found evidence for discrimination (97.1\%) and a large plurality (61.9\%) differentiated between taste-based versus statistical motives when discussing its source. However, only a small proportion (10.5\%) make the distinction between accurate and inaccurate beliefs, and a smaller fraction still (6.7\%) incorporates this distinction into the analysis. These data highlight that the economics literature has typically not considered inaccurate beliefs as a source of discrimination.

Next, we formally demonstrate how the possibility of inaccurate beliefs generates an identification problem for attempts to isolate the source of discrimination. We also show that failing to allow for mistaken beliefs can lead to a misclassification of belief-based discrimination as stemming from taste-based sources. We first distinguish between discrimination, which is a property of behavior, and partiality which is a primitive of the model (i.e. preferences and beliefs). In the context of the labor market, discrimination occurs when two workers generate identical signals but are evaluated differently based on their group identity. Partially refers to the source of this disparity based on model primitives: belief-based partiality corresponds to evaluators having different beliefs about decision-relevant attributes (e.g. productivity) depending on group identity, while preference-based partiality corresponds to evaluators setting different hiring thresholds based on group identity. The former is typically referred to as taste-based discrimination or prejudice, while the latter is referred to as statistical discrimination when beliefs about the distribution of the job-relevant factor are correct. We expand the standard approach by considering both accurate belief-based partiality (traditional statistical discrimination) and inaccurate belief-based partiality (inaccurate statistical discrimination).

To facilitate the analysis, we define isodiscrimination curves as the set of preferences and beliefs that lead to equivalent discrimination. Similar to indifference curves in utility theory, there is a collection of isodiscrimination curves that each correspond to a level of discrimination on a given set of signals. Notably, it is readily apparent that the same level of discrimination can stem from a continuum of preference and belief combinations. Isodiscrimination curves provide a tractable way to examine what a given data set can and cannot identify. 
We first show that collecting data on evaluation decisions, e.g. hiring, as a function of group identity and signals can be used to identify the isodiscrimination curve and rule out the case of no discrimination. Consider the correspondence study method, which uses an experimental technique where evaluators receive identical signals from people of different group identities. Perhaps the most famous example is the resume study by Bertrand and Mullainathan (2004), where experimenters sent out identical resumes to potential employeeswhere the only thing that differed was whether the group identity associated with the applicant's name - and looked at call-back rates as a measure of discrimination. Depending on the richness of the data, this technique can either identify an upper and lower bound on the isodiscrimination curve or the specific isodiscrimination curve. However, even if the latter case is possible, this method does not identify the source or even rule out any form of partiality. Let belief and preference partiality (neutrality) refer to an evaluator whose beliefs or preferences differ (do not differ) by group identity. In the case of the correspondence study method, for any evaluator with preference partiality and belief neutrality, there exists a continuum of evaluators with belief-based partiality and lower (or no) preference-based partiality that would exhibit the same level of observed discrimination. This includes beliefbased partiality about the mean of the relevant distribution, its variance, or the precision of signals of the relevant characteristic. In turn, additional data is necessary to isolate the source of discrimination.

In some cases, researchers have access to not only the evaluation decisions, but also to the underlying outcome distributions. In these cases, studies attempt to identify the source of discrimination through a technique often referred to as an outcomes-based test, which compares evaluation decisions to the true underlying distribution of the relevant characteristics for these decisions. This technique has been used to identify the source of discrimination in many domains, including lending, policing and bail decisions (Pope and Sydnor, 2011; Knowles, Persico, and Todd, 2001; Arnold, Dobbie, and Yang, 2018). For example, a researcher may compare differences in lending rates between two groups to differences in their loan default rates. Under the commonly-made assumption of accurate beliefs, we show that this technique isolates both the isodiscrimination curve and the exact source of discrimination. However, identification depends critically on the assumption that evaluators have accurate beliefs. Without this assumption, a continuum of preference and belief combinations can generate equivalent discrimination regardless of the underlying outcome distribution. As we formally demonstrate, the only case that can sometimes be ruled out with outcome data is accurate statistical discrimination, i.e. an evaluator with accurate beliefs and preference 
neutrality. ${ }^{2}$

Further, erroneously assuming that an evaluator has accurate beliefs when using the outcomes-based method leads a researcher to mistakenly attribute the share of discrimination arising from inaccurate beliefs to preferences. Depending on whether the inaccurate beliefs increase or decrease discrimination, the researcher will over- or underestimate the degree to which an evaluator has preferences that favor one of the groups. As an example of this identification issue, consider a study that measures productivity outcomes and finds evidence for discrimination in the treatment of two groups. If the researcher observes that both groups have identical distributions of productivity, she concludes that because accurate statistical discrimination cannot cannot explain behavior, the source of the observed discrimination must be preference-based. However, an alternative explanation is that evaluators have incorrect beliefs and use these beliefs to engage in inaccurate statistical discrimination. Without further data, it is impossible to distinguish between preference channels and incorrect beliefs. Alternatively, consider a study where discrimination is documented and the underlying distributions do differ. The study claims no evidence for prejudice as group differences can explain differential treatment. As we show, any departures from correct beliefs imply that prejudice does play a role in the observed discrimination.

Finally, we outline what type of data can be used to overcome the identification problem. One method, which we illustrate in Section 4, is to collect data on the subjective beliefs of evaluators. Combined with observing the evaluation decisions and signals, this allows for identification of preference and belief partiality. Data on the true outcome distributions is required to determine whether beliefs are accurate. Another method is varying the precision of information by increasing the number of signal draws supplied to evaluators. We demonstrate that this method can partially identify the source of discrimination: it identifies the extent of preference-based partiality, but cannot distinguish between different forms of belief-based partiality (i.e. differential means and variances of the relevant characteristic, or differential signal precisions). Notably, this method requires multiple signals from the same domain (e.g. number of positive reviews); if the multiple signals are from different domains (e.g. SAT scores and education history), then the identification problem persists.

We then use a stylized experimental setting to demonstrate the potential pitfalls of the identification problem and propose a portable method of addressing it. Participants are

\footnotetext{
${ }^{2}$ Other papers have highlighted identification challenges for outcomes-based tests, including the problem of infra-marginality (Ayres, 2002; Simoiu, Corbett-Davies, and Goel, 2017), as well as issues related to relying on administrative data that may condition on a post-treatment outcome (Knox, Lowe, and Mummolo, 2020). We raise a complementary concern that remains even if these other issues are solved.
} 
recruited to take part in a hiring experiment, where some are assigned the role of "worker" and others the role of "employer". Workers begin the first stage of the experiment by answering a series of questions. Employers are then shown profiles of 20 potential employees (workers from the initial stage) and asked the maximum wage they would be willing to pay to hire each. The profiles include a variety of worker-specific characteristics, such as their country of origin (US vs. India), gender, and age, interspersed amongst other information such as their beverage and movie preferences. Importantly, profiles do not include any information about performance on the question task. One worker profile is then selected. If the offered wage is above a randomly determined threshold, then the employer hires the worker - the worker earns a bonus and the employer is paid proportional to how many questions the worker answered correctly; if the offered wage is below the threshold, the worker is not hired.

We find that employers discriminate based on worker characteristics. Americans and females receive systematically lower wage offers than Indians and males. We find no evidence for age discrimination. According to the standard classification, the observed discrimination is generated by two potential sources. Employers may offer lower wages to American and female workers because they believe that members of those groups answer fewer questions correctly on average than Indian and male workers. Because they lack information on the productivity of any given worker, employers use these group statistics to inform their compensation decisions. Alternatively, employers may have animus towards members of the discriminated group and offer them lower wages because they do not want to reward them.

As discussed above, outcomes-based tests are often used to distinguish between statistical and taste-based discrimination by comparing the distributions of compensation decisions to the "ground truth" - the true distributions of performance across groups. If the difference in the group-specific performance distributions is similar to the difference in wages, then this is used as evidence that evaluators are statistically discriminating, i.e. they have belief-based partiality. Otherwise, discrimination is categorized as preference based. Our experiment allows us to measure the "ground truth" by comparing the number of questions answered correctly across the various groups. We find that Americans and Indians perform equally well on the task, while females perform less well than males. Under the assumption of accurate beliefs, we would conclude that the source of discrimination against Americans is preference based. Further, because the level of discrimination against women is substantially smaller than the actual gap in performance, this approach would conclude that there was preference based discrimination against men.

However, an alternative explanation is that individuals have no preference-based motives 
towards or against a particular group, but rather have inaccurate beliefs about the respective performance distributions. To identify this channel, we elicited the beliefs of employers and compared them to the "ground truth" distributions. Consistent with inaccurate statistical discrimination, employers mistakenly predicted that American workers perform much worse than their Indian counterparts, and that female workers only slightly underperform relative to males. Accounting for these inaccurate beliefs substantially changes the inferred source of discrimination. What was originally classified as preference-based discrimination in favor of Indians is mostly explained by mistaken beliefs - if anything, the preference-based channel goes against Indian workers. Similarly, a large portion of the gender gap in wages can be explained by inaccurate statistical discrimination.

The line between inaccurate beliefs and animus may sometimes be blurry. For example, individuals may develop inaccurate beliefs because they have animus against members of a particular group. We propose that these channels are separately identifiable through the provision of information. Specifically, if agents are provided with credible information on the relevant distributions, those with inaccurate beliefs should adjust their behavior accordingly. However, if mistaken beliefs merely mask an underlying animus, then agents are unlikely to change their behavior in response.

We implement this method in our experiment by providing employers with information on average performance by gender, nationality, and age. After receiving this information, participants were asked to make wage offers to 10 additional workers. We find that employers significantly changed their wage offers in the direction consistent with correcting their inaccurate beliefs. This methodology is portable outside of our stylized experimental setting as a way to identify animus-driven inaccurate beliefs.

The paper proceeds as follows. Section 2 presents a review of the economics literature on discrimination, demonstrating that very few papers consider mistaken beliefs when attempting to isolate its source. Section 3 formally outlines how a failure to account for the possibility of inaccurate beliefs leads to an identification problem. Section 4 illustrates a potential methodology for overcoming this identification problem through a stylized experiment. Section 5 concludes.

\section{Survey of the Literature}

We conducted a systematic survey of the economics literature on discrimination in order to determine: (1) how often papers seek to distinguish between taste-based and belief-based (statistical) sources of discrimination; (2) how often papers seek to distinguish between ac- 
curate and inaccurate beliefs for belief-based sources of discrimination. Table 1 tabulates the 105 papers published in 10 top economics journals between 1990 and 2018 that test for evidence of discrimination. Most papers that met our inclusion criteria (outlined below) found evidence of discrimination: 102 out of 105 papers, or $97.1 \%$ documented evidence for discrimination against at least one group that was considered in the paper. The majority of papers $(61.9 \%)$ discussed the source of discrimination as being driven by either preferences (taste-based) or beliefs (statistical), and nearly half of the papers (46.7\%) attempted to distinguish between these two sources through a formal test. However, very few papers even discussed the possibility that beliefs may be inaccurate (10.5\%), and fewer still examined whether beliefs were accurate or inaccurate $(6.7 \%){ }^{3}$ Despite the lack of discussion and explicit tests, we would argue that inaccurate statistical discrimination is a reasonable alternative to the interpretation chosen by the authors in nearly all of these cases.

We classified papers as "discuss taste-based versus statistical source" if preference versus belief-based motives for the documented discrimination were discussed in the text, and as "test for taste-based versus statistical source" if the paper either explicitly tested between different models of preference versus belief-based discrimination or implicitly tested the predictions of a belief-based model while taking the taste-based model as the null hypothesis. If a paper mentioned inaccurate or biased beliefs as a potential source of discrimination, it was classified as "discuss accurate versus inaccurate beliefs." Papers that tested whether inaccurate beliefs could be driving discrimination, either by directly eliciting beliefs or through other tests, were classified as "test for inaccurate beliefs." Finally, papers that elicited beliefs were classified as "measure beliefs." Three of the seven papers in this category did not test whether these elicited beliefs were accurate.

Method. In this section, we outline the method that we used to determine which papers to include in the survey and the data that we collected for each paper.

Inclusion Criteria. We focused on empirical papers published between 1990 and 2018 in the following journals: American Economic Journal: Applied, American Economic Journal: Policy, American Economic Review (excluding the Papers \& Proceedings issue), Econometrica, Journal of the European Economic Association, Journal of Labor Economics, Journal of Political Economy, the Quarterly Journal of Economics, Review of Economic Studies, and

\footnotetext{
${ }^{3}$ The papers that tested for inaccurate beliefs include List (2004); Hedegaard and Tyran (2018); Mobius and Rosenblat (2006); Fershtman and Gneezy (2001); Arnold et al. (2018); Agan and Starr (2017). Beaman, Chattopadhyay, Duflo, Pande, and Topalova (2009) use the Implicit Association Test (IAT) to elicit a 'taste' against female politicians, though the authors also interpret IAT scores as a measure of implicit beliefs. We include this paper on the list as well.
} 
Table 1. Summary of Literature Review on Discrimination

\begin{tabular}{lcccc}
\hline & All: $\mathbf{1 9 9 0}-\mathbf{2 0 1 8}$ & \multicolumn{2}{c}{ Recent: 2014 - 2018 } \\
& \# Papers & \% Total & \# Papers & \% Total \\
\hline Papers meeting inclusion criteria & 105 & $100.0 \%$ & 31 & $100.0 \%$ \\
Evidence of discrimination & 102 & $97.1 \%$ & 31 & $100.0 \%$ \\
Discuss taste-based versus statistical source & 65 & $61.9 \%$ & 23 & $74.2 \%$ \\
Test for taste-based versus statistical source & 49 & $46.7 \%$ & 16 & $51.6 \%$ \\
Discuss accurate versus inaccurate beliefs & 11 & $10.5 \%$ & 5 & $16.1 \%$ \\
Test for inaccurate beliefs & 7 & $6.7 \%$ & 3 & $9.7 \%$ \\
Measure beliefs & 7 & $6.7 \%$ & 3 & $9.7 \%$ \\
\hline
\end{tabular}

Review of Economics and Statistics. We acknowledge that the economics literature on discrimination includes important contributions from other journals. We restricted attention to these ten journals as a representative sample in order for the scope of the survey to include a manageable number of papers.

We proceeded in two steps to determine whether to include a paper published in the relevant time frame and journals. First, in each journal, we searched for all empirical papers that had at least one of the following search terms in the title:

$$
\begin{gathered}
\{\text { discrimination, prejudice, bias, biases, biased, disparity, disparities, stereotype, } \\
\text { stereotypes, premium }\}
\end{gathered}
$$

or at least one of the following search terms in the abstract:

$$
\text { \{discrimination, prejudice\} }
$$

or at least one of the search terms from each of the following lists in the abstract:

$$
\begin{gathered}
\{\text { racial, race, gender, sex, ethnic, religious, beauty }\} \\
\text { and } \\
\{\text { bias, biased, disparity, stereotype, stereotypes, premium\}. }
\end{gathered}
$$

Second, we restricted attention to papers that attempted to causally document differential treatment of individuals based on their group identity. This eliminated papers on unrelated topics, including the industrial organization literature on price discrimination, the financial literature on the risk premium, theoretical models, and the experimental literature that documents behavioral differences such as gender differences in risk preferences. ${ }^{4}$

\footnotetext{
${ }^{4}$ We also excluded some papers that met our objective criteria but which we viewed as not relevant to
} 
Table 2. Publications by Journal and Decade

\begin{tabular}{lcccc}
\hline & \multicolumn{4}{c}{ Number of Papers } \\
& $\mathbf{1 9 9 0 - 9 9}$ & $\mathbf{2 0 0 0 - 0 9}$ & $\mathbf{2 0 1 0 - 2 0 1 8}$ & Total \\
\hline AEJ: Applied & 0 & 1 & 7 & 8 \\
AEJ: Policy & 0 & 0 & 2 & 2 \\
AER & 4 & 7 & 6 & 17 \\
EMA & 0 & 0 & 0 & 0 \\
JEEA & 0 & 1 & 1 & 2 \\
JLE & 2 & 8 & 12 & 22 \\
JPE & 2 & 6 & 1 & 9 \\
ReStud & 1 & 2 & 3 & 6 \\
ReStat & 5 & 6 & 11 & 22 \\
QJE & 4 & 4 & 9 & 17 \\
\hline Total & $\mathbf{1 8}$ & $\mathbf{3 5}$ & $\mathbf{5 2}$ & $\mathbf{1 0 5}$ \\
\hline
\end{tabular}

Data Collection. For each paper that met our inclusion criteria, we recorded the following information: data source (laboratory experiment, field experiment, audit/correspondence study, observational data study, other), empirical method (reduced form analysis, structural analysis), group identity of interest (race, gender, ethnicity, religion, sexuality, class/income, other), domain of study (labor market, legal, education, financial, consumer purchases - nonfinancial, evaluations, other), measure of discrimination (i.e. difference in call back rates), whether the paper distinguishes between taste-based and statistical discrimination, whether the paper distinguishes between accurate and inaccurate statistical discrimination, whether discrimination was documented, whether the study identified the source of discrimination, and whether the study measured beliefs about an individual's predicted attribute by group identity.

Summary Statistics. We found 105 papers that met our inclusion criteria. Table 2 lists the number of papers broken down by journal and decade of publication. The full list of papers is included in the Online Appendix. Out of the papers surveyed, 11 conducted audit or correspondence studies, 7 conducted another type of field experiment, 3 conducted a laboratory experiment and 84 analyzed observational data.

Discrimination was studied for a variety of group identities and in a variety of domains.

the spirit of the exercise. More specifically, we excluded papers that could not be classified as either a "Yes" or "No" for the criteria outlined in Table 1. For example, Gneezy, Niederle, and Rustichini (2003) examine behavioral differences between men and women but do not study discrimination per se. Similarly, Cameron and Heckman (2001) examine the extent to which the racial and ethnic gap in college attendance can be explained by long-run versus short-run factors but do not address discrimination. 
Table 3. Type and Domain of Discrimination

\begin{tabular}{lccc}
\hline & $\begin{array}{c}\text { All Papers } \\
\text { \# Papers }\end{array}$ & $\begin{array}{c}\text { Evidence of Discrimination } \\
\text { \# Papers }\end{array}$ & \% Total \\
\hline Group Identity & 58 & 56 & $96.6 \%$ \\
Race & 37 & 35 & $94.6 \%$ \\
Gender & 6 & 6 & $100.0 \%$ \\
Ethnicity & 1 & 1 & $100.0 \%$ \\
Religion & 1 & 1 & $100.0 \%$ \\
Sexuality & 1 & 1 & $100.0 \%$ \\
Class/Income & 7 & 7 & $100.0 \%$ \\
Physical Traits / Appearance & 5 & 5 & $100.0 \%$ \\
Other & & & \\
Domain & & & \\
Labor Market & 58 & 57 & $98.3 \%$ \\
Legal & 12 & 12 & $100.0 \%$ \\
Education & 9 & 9 & $100.0 \%$ \\
Financial & 5 & 4 & $80.0 \%$ \\
Consumer Markets (not financial) & 6 & 6 & $100.0 \%$ \\
Other & 17 & 16 & $94.1 \%$ \\
\hline
\end{tabular}

The most frequent group identities were race (58 papers) and gender (37 papers), followed by physical traits / appearance ( 7 papers) and ethnicity (6 papers). The most frequent domain was labor markets (58 papers), followed by legal contexts (12 papers), education (9 papers), non-financial consumer markets (6 papers) and financial markets ( 5 papers). Table 3 summarizes the papers by group identity and domain. Some papers in the survey studied multiple group identities or domains; therefore, some papers are counted in multiple rows of the table.

\section{A Model of Discrimination with Inaccurate Beliefs}

In this section, we develop a general model of discrimination with inaccurate beliefs. An evaluator learns about a worker's productivity from group identity and a signal, then decides whether to hire the worker. Inaccurate beliefs refer to the possibility that the evaluator misperceives key population statistics, including how the productivity and signal distributions vary by group. We show that an identification problem arises when seeking to determine the source of discrimination: namely, many different combinations of evaluator preferences and beliefs lead to the same patterns of discrimination. Further, we show that maintaining the assumption of accurate beliefs when it does not hold can lead a researcher to mistakenly 
classify discrimination as arising from preferences when it is actually driven by beliefs. Finally, we demonstrate that two methods - eliciting beliefs and manipulating information can at least partially separate whether discrimination stems from preferences or beliefs.

\subsection{Set-up}

Worker. Consider a worker who has observable group identity $g \in\{M, F\}$ and unobservable productivity a drawn from normal distribution $N\left(\mu_{g}, 1 / \tau_{g}\right)$, with mean $\mu_{g} \in \mathbb{R}$ and concentration $\tau_{g}>0$. The worker completes a task, such as an interview or test, that generates a signal of productivity $s=a+\epsilon$, where $\epsilon \sim N\left(0,1 / \eta_{g}\right)$ with precision $\eta_{g}>0$. Without loss of generality, we focus on discrimination against workers from group $F$.

Evaluator. An evaluator decides whether to hire the worker, $v \in\{0,1\}$ where 1 corresponds to hire and 0 corresponds to do not hire. Before making this decision, the evaluator observes the worker's group identity $g$ and signal $s$. The evaluator holds subjective beliefs $\hat{\mu}_{g} \in \mathbb{R}$ and $\hat{\tau}_{g}>0$ about the mean and concentration of productivity for group $g$, and subjective belief $\hat{\eta}_{g}>0$ about the precision of the signal for group $g$. We allow for the possibility that the evaluator has a misspecified model of the signal or productivity distribution, in that her subjective distributions differ from the true distributions. ${ }^{5}$

Given these subjective distributions, the evaluator uses Bayes rule to update her belief about the worker's productivity. She hires the worker if her subjective posterior belief about expected productivity is above a group-specific hiring threshold $u_{g} \in \mathbb{R}$. This hiring threshold is a reduced form representation of how the evaluator's payoff depends on productivity and group identity. ${ }^{6}$ We refer to the evaluator's preferences as represented by hiring threshold $u_{g}$ and subjective beliefs $\left(\hat{\mu}_{g}, \hat{\tau}_{g}, \hat{\eta}_{g}\right)$ for each group $g \in\{M, F\}$ as her type, denoted by $\theta$. Given type $\theta$, let $v(s, g, \theta) \equiv \mathbb{1}\left\{\hat{E}_{\theta}[a \mid s, g] \geq u_{g}\right\}$ denote the optimal hiring decision for a worker from group $g$ who generates signal $s$, where $\hat{E}_{\theta}$ denotes the expectation taken with respect to type $\theta$ 's subjective beliefs.

\footnotetext{
${ }^{5}$ An additional form of misspecification that we do not discuss is the possibility that an evaluator believes that the mean of the signal differs by group identity. For example, all signals for group $F$ are inflated by a constant $b>0$ i.e. $s=a+b+\epsilon$, and therefore, the evaluator discounts a signal to $s-b$ for group $F$.

${ }^{6}$ The microfoundation for this reduced form is as follows. If the evaluator hires the worker, she earns a payoff that is linear in productivity and also depends on group identity, $m_{g} a+b_{g}$, where $m_{g}>0$ is a groupspecific marginal value of productivity and $b_{g} \in \mathbb{R}$ is a group-specific taste parameter. If she does not hire the worker, she earns outside option $\underline{u}$. The evaluator maximizes her expected payoff. She hires the worker if and only if $\hat{E}\left[m_{g} a+b_{g} \mid s, g\right]>\underline{u}$, or $\hat{E}[a \mid s, g]>\left(\underline{u}-b_{g}\right) / m_{g} \equiv u_{g}$, where $\hat{E}$ denotes the expectation with respect to the evaluator's subjective beliefs. Therefore, $u_{g}$ is a reduced form representation of the evaluator's payoff.
} 
Discrimination. The causal effect of group identity on hiring is captured by the difference between the hiring decision for a worker from group $M$ versus $F$, holding fixed the signal of productivity. Let $D(s, \theta) \equiv v(s, M, \theta)-v(s, F, \theta)$ denote the difference between the hiring decision an evaluator of type $\theta$ makes for members of group $M$ and $F$ after observing signal s. Discrimination occurs when two workers who generate identical signals are evaluated differently based on their group identity, $D(s, \theta) \neq 0$; it occurs against group $F$ if $D(s, \theta)>0$ and against group $M$ if $D(s, \theta)<0$. We say that discrimination exists if there is an $s$ such that $D(s, \theta) \neq 0$ and there is no discrimination if $D(s, \theta)=0$ for all $s \in \mathbb{R}$. We are interested in when different sets of beliefs and preferences give rise to the same discriminatory behavior, which we refer to as equivalent discrimination.

Definition 1 (Equivalent Discrimination). Two evaluators of types $\theta$ and $\theta^{\prime}$ exhibit equivalent discrimination if $D(s, \theta)=D\left(s, \theta^{\prime}\right)$ for all $s \in \mathbb{R}$.

Partiality. We next categorize different forms of preferences and beliefs. We use the term partiality to refer to properties of these model primitives in order to distinguish them from discrimination, which is a property of behavior and a consequence of said primitives. An evaluator with preference partiality against group $F$ sets a higher threshold for hiring workers from group $F$ relative to workers from group $M$. This leads her to make different hiring decisions even if she has the same belief about the productivity of a worker from each group.

Definition 2 (Preference Partiality). An evaluator has preference partiality if $u_{F} \neq u_{M}$ and preference neutrality if $u_{F}=u_{M}$.

An evaluator with belief partiality has different subjective posterior distributions of productivity for each group. This difference can stem from differential beliefs about average productivity, the concentration of productivity, the signal precision, or a combination thereof. Belief partiality is inaccurate if the subjective beliefs differ from the true distribution parameters.

Definition 3 (Belief Partiality). An evaluator has belief partiality if $\left(\hat{\mu}_{F}, \hat{\tau}_{F}, \hat{\eta}_{F}\right) \neq\left(\hat{\mu}_{M}, \hat{\tau}_{M}, \hat{\eta}_{M}\right)$ and belief neutrality if $\left(\hat{\mu}_{F}, \hat{\tau}_{F}, \hat{\eta}_{F}\right)=\left(\hat{\mu}_{M}, \hat{\tau}_{M}, \hat{\eta}_{M}\right)$. This belief partiality stems from (i) lower expected productivity if $\hat{\mu}_{F}<\hat{\mu}_{M}$; (ii) lower (higher) concentration if $\hat{\tau}_{F}<\hat{\tau}_{M}\left(\hat{\tau}_{F}>\hat{\tau}_{M}\right)$; and (iii) lower (higher) signal precision if $\hat{\eta}_{F}<\hat{\eta}_{M}\left(\hat{\eta}_{F}>\hat{\eta}_{M}\right)$. Belief partiality is accurate if $\left(\hat{\mu}_{g}, \hat{\tau}_{g}, \hat{\eta}_{g}\right)=\left(\mu_{g}, \tau_{g}, \eta_{g}\right)$ for $g \in\{M, F\}$ and otherwise is inaccurate.

Using this terminology, what the literature often refers to as taste-based discrimination corresponds to differential treatment stemming from preference partiality, while what is often referred to as statistical discrimination corresponds to differential treatment stemming from belief partiality. 
Markets. Adopting terminology from Bartoš, Bauer, Chytilová, and Matějka (2016), we define several types of markets relevant for our analysis. An evaluator believes the market is lemon-dropping for a group if the hiring threshold is low relative to perceived average productivity, $u_{g}<\hat{\mu}_{g}$. In this case, the evaluator wants to hire a worker from group $g$ in the absence of a signal. The evaluator believes the market is cherry-picking for a group if the hiring threshold is high relative to average productivity, $\hat{\mu}_{g}<u_{g}$. In this case, the evaluator does not want to hire a worker from group $g$ in the absence of a signal. A market is mixed if the evaluator believes it is cherry-picking for one group and lemon-dropping for the other group.

Discussion of Model. We focus on binary evaluations for a population of workers with normally distributed productivity and signals. These assumptions are for tractability: the simple set-up allows us to illustrate how inaccurate beliefs impact discrimination in a clear, focused way. Our main insights are conceptually robust, in that they will also arise for other forms of evaluations - for example, when a worker receives a wage offer or a rating selected from an interval - and for other distributions of population characteristics or signals.

\subsection{Equivalent Discrimination and Isodiscrimination Curves}

We first derive the sets of beliefs and preferences that give rise to equivalent discrimination. Given signal $s$ and group identity $g$, the evaluator's posterior belief about productivity is normally distributed with mean $\hat{\mu}_{g}(s, \theta) \equiv\left(\hat{\tau}_{g} \hat{\mu}_{g}+\hat{\eta}_{g} s\right) /\left(\hat{\tau}_{g}+\hat{\eta}_{g}\right)$ and variance $1 /\left(\hat{\tau}_{g}+\hat{\eta}_{g}\right)$. Since the posterior mean is monotonic with respect to $s$, the optimal hiring decision can be represented as a cut-off rule with respect to the signal.

Lemma 1 (Hiring Signal Threshold). A type $\theta$ evaluator hires a worker from group $g$, $v(s, g, \theta)=1$, if and only if the worker generates a signal

$$
s \geq \bar{s}(\theta, g) \equiv\left(\frac{\hat{\tau}_{g}+\hat{\eta}_{g}}{\hat{\eta}_{g}}\right) u_{g}-\frac{\hat{\tau}_{g}}{\hat{\eta}_{g}} \hat{\mu}_{g}
$$

The signal required to hire a worker is increasing in the evaluator's group-specific hiring threshold and decreasing in the prior belief about average productivity. In a cherry-picking market, this signal is increasing in the concentration of productivity - intuitively, concentration of productivity is undesirable when the evaluator is trying to select workers in the top tail of the distribution. In contrast, it is decreasing in the signal precision - a precise signal is beneficial to the worker when the evaluator would not hire a worker in the absence of a signal. These comparative statics reverse in a lemon-dropping market: concentration of 
productivity is desirable - thereby lowering the bar - when the evaluator is trying to avoid workers in the bottom tail of the distribution and a precise signal is detrimental to the worker - raising the bar - when the evaluator would hire a worker in the absence of a signal.

From Lemma 1, an evaluator of type $\theta$ exhibits discrimination against group $F$ if she sets a higher signal threshold for group $F, \bar{s}(\theta, F)>\bar{s}(\theta, M)$. When this is the case, discrimination occurs on the interval of signals that lie between the two hiring thresholds, $s \in[\bar{s}(\theta, M), \bar{s}(\theta, F))$. Therefore, if two types discriminate, then they exhibit equivalent discrimination when they have preferences and beliefs that lead to the same signal thresholds for each group. We use this observation and Eq. (1) to characterize isodiscrimination curves - that is, the sets of preferences and beliefs that lead to equivalent discrimination.

Proposition 1 (Equivalent Discrimination). For any constants $\left(s_{M}, s_{F}\right) \in \mathbb{R}^{2}$ with $s_{M} \neq s_{F}$, equivalent discrimination occurs for the set of types that have signal threshold $s_{M}$ for group $M$ and $s_{F}$ for group $F$,

$$
\text { Isodiscrimination Curve }=\left\{\begin{array}{l|l}
\left(u_{g}, \hat{\mu}_{g}, \hat{\tau}_{g}, \hat{\eta}_{g}\right)_{g \in\{M, F\}} & \begin{array}{l}
\frac{\hat{\tau}_{M}+\hat{\eta}_{M}}{\hat{\eta}_{M}} u_{M}-\frac{\hat{\tau}_{M}}{\hat{\eta}_{M}} \hat{\mu}_{M}=s_{M} \\
\frac{\hat{\tau}_{F}+\hat{\eta}_{F}}{\hat{\eta}_{F}} u_{F}-\frac{\hat{\tau}_{F}}{\hat{\eta}_{F}} \hat{\mu}_{F}=s_{F}
\end{array}
\end{array}\right\},
$$

while the isodiscrimination curve corresponding to no discrimination is characterized by the set of types that satisfy Eq. (2) for each $s_{M}=s_{F}$.

Similar to indifference curves in utility theory, there are a collection of isodiscrimination curves, each one corresponding to a different level of discrimination.

Fig. 1 illustrates isodiscrimination curves in two-dimensions. Each curve plots the set of preferences and perceived average productivities for group $F$ that lead to a given level of discrimination, holding fixed the other parameters. As illustrated in the figure, there are a continuum of evaluator types that exhibit equivalent discrimination. For example, an evaluator with mild preference partiality and extreme belief partiality will exhibit equivalent discrimination to an evaluator with more extreme preference partiality and mild belief partiality. The red line traces out the isodiscrimination curve of no discrimination. Isodiscrimination curves above this line correspond to discrimination against group $F$, while isodiscrimination curves below this line correspond to discrimination against group $M$. Moving northwest from the line of no discrimination, i.e. increasing the hiring threshold and decreasing perceived average productivity, results in discrimination on a larger set of signals. The blue dotted line traces out preference neutrality (i.e. $u_{F}=u_{M}$ ) and the green dotted line traces out belief 


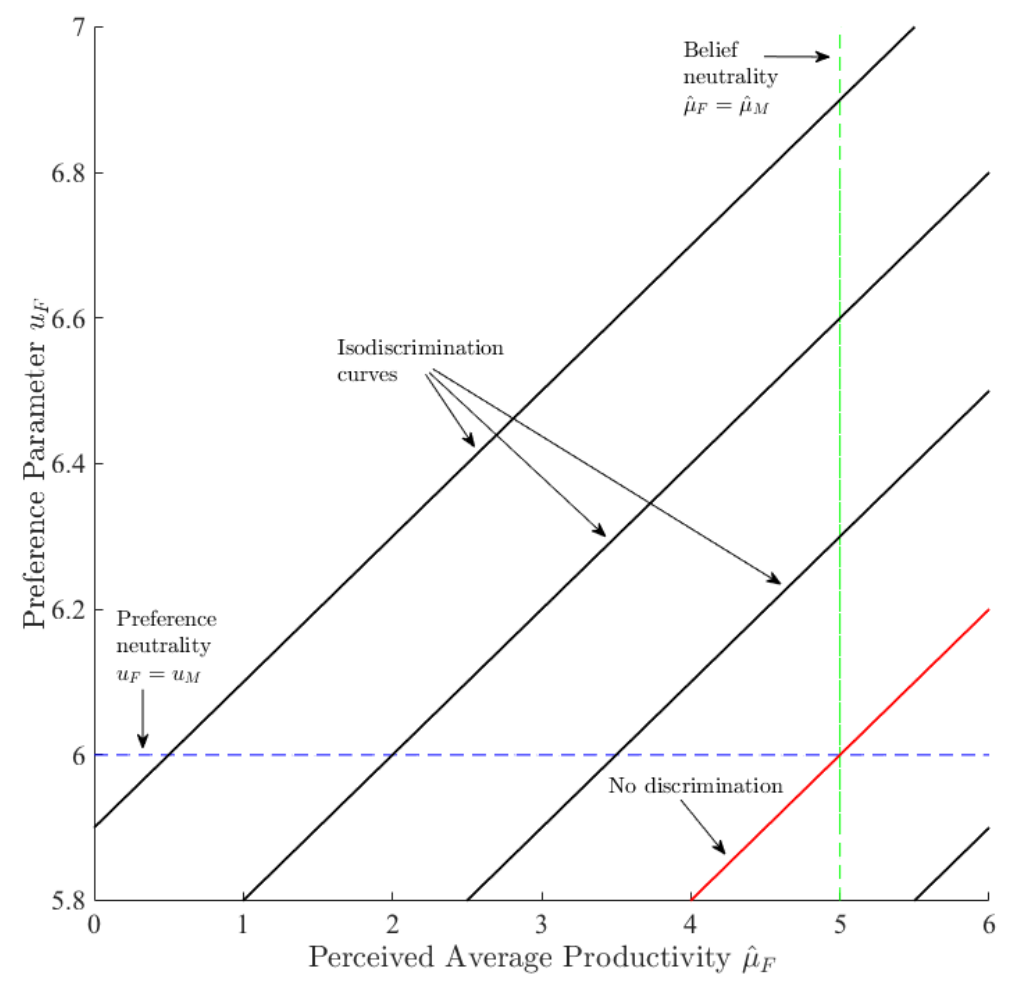

Figure 1. Isodiscrimination Curves

$\left(u_{M}, \hat{\mu}_{M}, \hat{\tau}_{M}, \hat{\eta}_{M}\right)=(6,5, .5,2),\left(\hat{\tau}_{F}, \hat{\eta}_{F}\right)=(.5,2)$

neutrality (i.e. $\hat{\mu}_{F}=\hat{\mu}_{M}$ ). The quadrant above and to the left of these lines corresponds to the region in which there is preference and belief partiality against group $F$, the quadrant below and to the left corresponds to the region in which there is belief partiality against group $F$ and preference partiality in favor of group $F$, while the opposite holds for the quadrant above and to the right. As can be seen in the figure, a given level of discrimination can stem from both a higher hiring threshold and lower perceived average productivity for group $F$, lower perceived average productivity that is somewhat offset by a more favorable hiring threshold, or vice versa. Finally, the quadrant below and to the right of the neutrality lines corresponds to the region in which there is preference and belief partiality against group $M$. In this region, it is not possible for there to be discrimination against group $F$.

\subsection{Identifying Discrimination.}

We next explore which model primitives are identified in common empirical designs used to study discrimination. To this end, a property of the model is identified if there exists 
an injective relationship between the observed data and the property (Haavelmo, 1944). Informally, this means that the property of interest can be backed out from available data. Throughout this section, we assume that at the minimum, a researcher observes the group identity $g$ and hiring decision $v$ for each worker.

Existence and Isodiscrimination Curves. Establishing the existence of discrimination against group $F$ corresponds to showing that there exists an $s$ such that $D(s, \theta)>0$. If the researcher also observes the signal $s$ for each worker, then it is straightforward to identify existence by comparing the hiring decisions for each group across a sufficiently rich set of workers to ensure that the data includes signals in the interval where discrimination occurs. In practice, this direct method is generally not possible. An alternative method is a correspondence study, which randomly assigns group identity and signals to a set of fictitious workers, then elicits hiring decisions (for example, the classic resume study of Bertrand and Mullainathan (2004)). ${ }^{7}$ This ensures that workers from each group in the fictitious sample have the same distribution over signals, and therefore, any differences in hiring can be causally attributed to group identity. Provided there is a sufficiently rich set of signals so that there is at least one signal $s \in[\bar{s}(\theta, M), \bar{s}(\theta, F)$ ), this method identifies the existence of discrimination. ${ }^{8}$ A correspondence study can also identify $D(s, \theta)$ if it is possible to create fictitious workers who are identical aside from assigned group identity. In this case, if hiring decisions are observed for a sufficiently rich set of signals for each group, then the signal thresholds, and therefore, the isodiscrimination curve, are identified.

Observation 1 (Identifying Isodiscrimination Curve). Observing $v, g$, and $s$ for a continuum of workers in each group with signals in an interval that includes the signal thresholds identifies the isodiscrimination curve.

Otherwise, an upper and lower bound for the signal thresholds, and therefore, a set of isodiscrimination curves, are identified.

Source of Discrimination. While the direct or correspondence study methods are effective for identifying the existence of discrimination and the isodiscrimination curve, researchers are often interested in identifying the source of discrimination i.e. the form of partiality that generates the observed discriminatory behavior. It is well known that cor-

\footnotetext{
${ }^{7}$ An audit study uses a similar randomized procedure to identify discrimination. Here, experimental confederates with different group identities interact with evaluators while following the same script. Different treatment of the confederates based on group identity is classified as discrimination.

${ }^{8}$ Note that failing to identify the existence of discrimination on a subset of the signal space does not establish that there is no discrimination, i.e. $D(s, \theta)=0$ for all $s$.
} 
respondence studies cannot distinguish between discrimination stemming from preference partiality and accurate belief partiality (see for example Bertrand and Mullainathan 2004) and similarly, neither can the direct method. The same insight extends to inaccurate belief partiality.

To formalize this insight, we show that for any evaluator type, there exist evaluator types with a single category of preference or belief partiality that exhibit equivalent discrimination. This establishes that each form of partiality in isolation can generate a given pattern of discrimination - in other words, each isodiscrimination curve intersects the lines of preference neutrality and each form of belief neutrality. Therefore, identifying the isodiscrimination curve does not identify the source of discrimination or even rule out any of the potential sources.

Proposition 2 (Equivalent Sources). For any type $\theta=\left(u_{g}, \hat{\mu}_{g}, \hat{\tau}_{g}, \hat{\eta}_{g}\right)_{g \in\{M, F\}}$ that discriminates against group $F$, there are a continuum of types that exhibit equivalent discrimination, including:

1. A type $\theta^{\prime}$ with preference partiality against group $F$ and belief neutrality, $u_{F}^{\prime}>u_{M}^{\prime}$ and $\left(\hat{\mu}_{F}^{\prime}, \hat{\tau}_{F}^{\prime}, \hat{\eta}_{F}^{\prime}\right)=\left(\hat{\mu}_{M}^{\prime}, \hat{\tau}_{M}^{\prime}, \hat{\eta}_{M}^{\prime}\right)$;

2. A type $\theta^{\prime}$ with preference neutrality and belief partiality due to lower expected productivity, $\hat{\mu}_{F}^{\prime}<\hat{\mu}_{M}^{\prime}$ and $\left(u_{F}^{\prime}, \hat{\tau}_{F}^{\prime}, \hat{\eta}_{F}^{\prime}\right)=\left(u_{M}^{\prime}, \hat{\tau}_{M}^{\prime}, \hat{\eta}_{M}^{\prime}\right)$;

3. A type $\theta^{\prime}$ that believes the market is cherry-picking (lemon-dropping) and has preference neutrality and belief partiality due to higher (lower) concentration of productivity, $\hat{\tau}_{F}^{\prime}>$ $\hat{\tau}_{M}^{\prime}\left(\hat{\tau}_{F}^{\prime}<\hat{\tau}_{M}^{\prime}\right)$ and $\left(u_{F}^{\prime}, \hat{\mu}_{F}^{\prime}, \hat{\eta}_{F}^{\prime}\right)=\left(u_{M}^{\prime}, \hat{\mu}_{M}^{\prime}, \hat{\eta}_{M}^{\prime}\right)$;

4. A type $\theta^{\prime}$ that believes the market is cherry-picking (lemon-dropping) and has preference neutrality and belief partiality due to lower (higher) signal precision, $\hat{\eta}_{F}^{\prime}<\hat{\eta}_{M}^{\prime}\left(\hat{\eta}_{F}^{\prime}>\hat{\eta}_{M}^{\prime}\right)$ and $\left(u_{F}^{\prime}, \hat{\mu}_{F}^{\prime}, \hat{\tau}_{F}^{\prime}\right)=\left(u_{M}^{\prime}, \hat{\mu}_{M}^{\prime}, \hat{\tau}_{M}^{\prime}\right)$.

Given a level of discrimination against group $F$, a higher preference parameter for group $F$ than group $M$ or a lower perceived average productivity generates the observed discrimination. In the cases of perceived concentration of productivity or signal precision, the direction of partiality that leads to discrimination against group $F$ depends on the type of market: a higher perceived concentration of productivity or lower signal precision generate discrimination in a cherry-picking market, while the opposite holds in a lemon-dropping market. This stems from the comparative static for the parameter of interest in Eq. (1): as discussed following Lemma 1, how these parameters impact the signal thresholds, and therefore, the level of discrimination, depends on the type of market. 


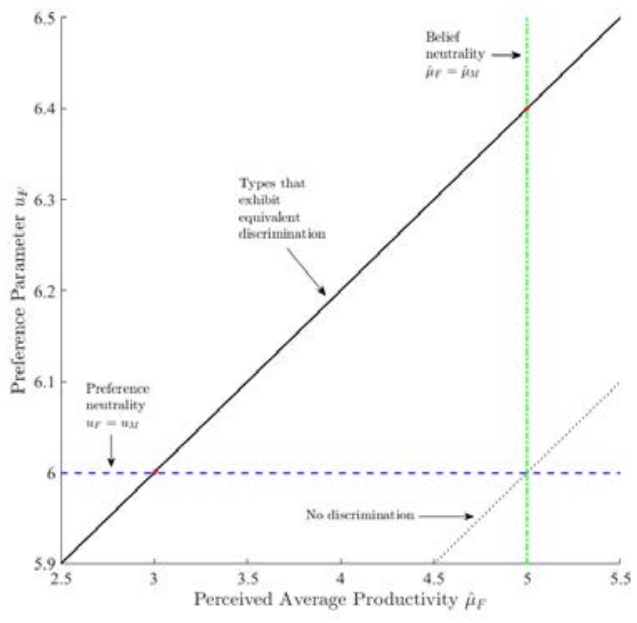

(a) Preference + perceived average productivity pairs; $\left(\hat{\tau}_{F}, \hat{\eta}_{F}\right)=(.5,2)$

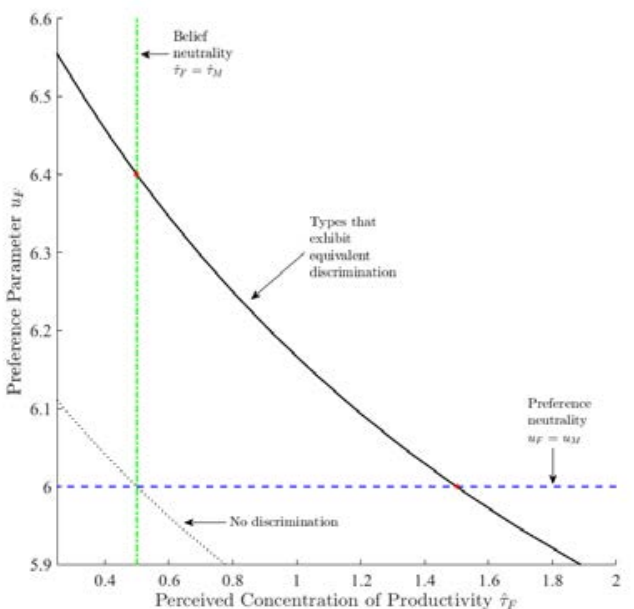

(b) Preference + perceived concentration of productivity pairs; $\left(\hat{\mu}_{F}, \hat{\eta}_{F}\right)=(5,2)$

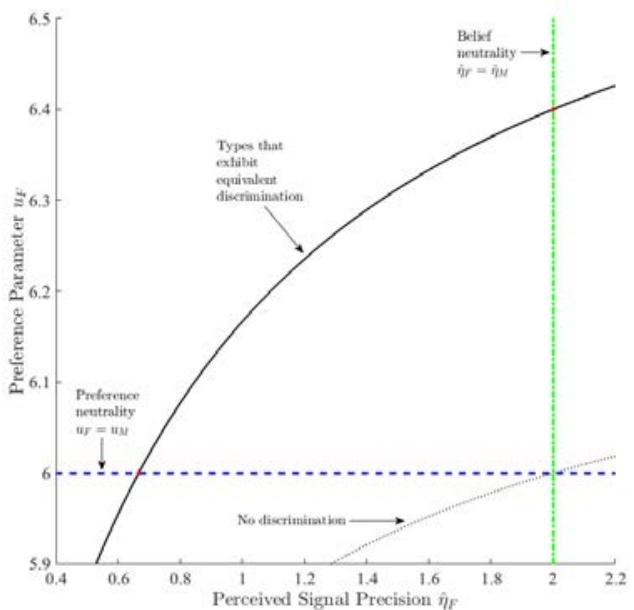

(c) Preference + perceived signal precision pairs; $\left(\hat{\mu}_{F}, \hat{\tau}_{F}\right)=(5, .5)$

Figure 2. Equivalent Sources

Isodiscrimination curve $\left(s_{M}, s_{F}\right)=(6.25,6.75) ;\left(u_{M}, \hat{\mu}_{M}, \hat{\tau}_{M}, \hat{\eta}_{M}\right)=(6,5, .5,2)$

Fig. 2 illustrates each evaluator type constructed in Proposition 2. Fixing an evaluator type that results in signal cut-offs $s_{M}=6.25$ and $s_{F}=6.75$, the first panel illustrates the continuum of preference parameters and perceived average productivity for group $F$ that exhibit equivalent discrimination. This includes the type with belief neutrality described in part (i), denoted by the asterisk where the isodiscrimination curve intersects the dotted line of belief neutrality, and the type with preference neutrality described in part (ii), denoted 
by the asterisk where the isodiscrimination curve intersects the dotted line of preference neutrality. The second panel repeats this exercise for the continuum of preference parameters and perceived concentrations of productivity, illustrating the types described in parts (i) and (iii), while the third panel does so for the continuum of preference parameters and perceived signal precisions, illustrating the types described in parts (i) and (iv).

Given Proposition 2, we see that even if it is possible to identify the isodiscrimination curve, it is not possible to identify the source of discrimination, or even rule out some of the potential sources.

Corollary 1. Identifying the isodiscrimination curve does not rule out preference partiality or belief partiality due to differential perceived average productivity, concentration of productivity, or signal precisions.

Therefore, additional data is necessary in order to separate these sources.

Outcomes-based Test. A common method to identify the source of discrimination is to compare evaluations to the outcome distribution for each group. In the current framework, this corresponds to comparing hiring decisions to the productivity and signal distributions. We next show that this method crucially relies on the assumption of accurate beliefs: when one allows for the possibility of inaccurate beliefs, the source can no longer be identified.

When implementing an outcomes-based test, the literature typically assumes accurate beliefs. Under this assumption, observing differential treatment and identical distributions implies preference partiality, whereas observing different distributions suggests (accurate) belief partiality (potentially coupled with preference partiality). In either case, the evaluator's type is identified.

Observation 2 (Accurate Beliefs: Identification of Source). Assume an evaluator has accurate beliefs and suppose a researcher can identify the isodiscrimination curve, i.e. from observing $v, g$, and $s$. Observing $\left(\mu_{g}, \tau_{g}, \eta_{g}\right)$ for $g \in\{M, F\}$ identifies the evaluator's type, and therefore, the exact source(s) of discrimination.

Fig. 3 illustrates how the assumption of accurate beliefs identifies the evaluator's type specifically, the unique preference parameter that is consistent with the observed isodiscrimination curve. In this example, observing the productivity and signal distributions identifies $u_{F}=6.3$ and $u_{M}=6$. Given $\mu_{F}=4.5$ and $\mu_{M}=5$, the evaluator has both preference partiality and accurate belief partiality.

Identification crucially depends on the assumption that beliefs are accurate. When beliefs may be inaccurate, then observing the productivity and signal distributions does not allow 


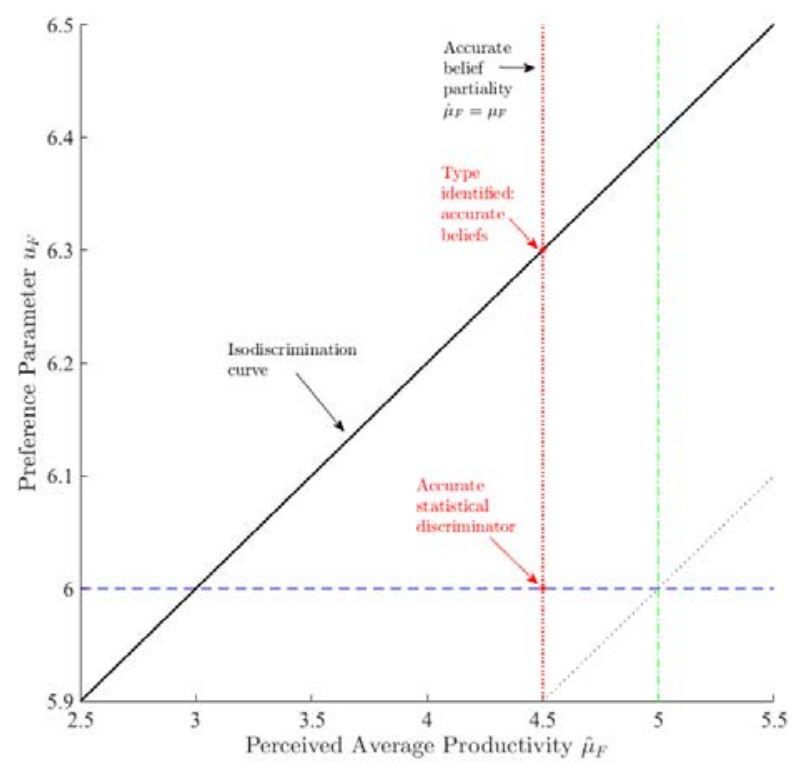

Figure 3. Outcomes-based Test

Isodiscrimination curve $\left(s_{M}, s_{F}\right)=(6.25,6.75)$; true distributions $\left(\mu_{M}, \tau_{M}, \eta_{M}\right)=(5, .5,2)$, $\left(\mu_{F}, \tau_{F}, \eta_{F}\right)=(4.5, .5,2) ;\left(u_{M}, \hat{\mu}_{M}, \hat{\tau}_{M}, \hat{\eta}_{M}\right)=(6,5, .5,2)$; blue dotted line: preference neutrality $\left(u_{F}=u_{M}\right)$; green dotted line: belief neutrality $\left(\hat{\mu}_{F}=\hat{\mu}_{M}\right)$; black dotted line: no discrimination.

a researcher to separate preference partiality from inaccurate belief partiality. Specifically, when the distributions are the same, differential treatment could be due to preferences, inaccurate beliefs, or a combination of the two. Similarly, when the distributions differ, differential treatment could stem from accurate beliefs or inaccurate beliefs coupled with preference partiality.

Observation 3 (Inaccurate Beliefs: An Identification Failure). Suppose a researcher can identify the isodiscrimination curve, i.e. from observing $v, g$, and s. Observing $\left(\mu_{g}, \tau_{g}, \eta_{g}\right)$ for $g \in\{M, F\}$ does not identify the evaluator's type.

In Fig. 3, any combination of preferences and beliefs on the isodiscrimination curve generate equivalent discrimination, regardless of the true average productivity.

Taken together, Observations 2 and 3 establish an important insight: erroneously assuming that an evaluator has accurate beliefs leads a researcher to mistakenly attribute the share of discrimination arising from inaccurate beliefs to preferences. To formalize this insight, we first define how to isolate the component of discrimination that is due to inaccurate beliefs.

Definition 4. Given a type $\theta$ with inaccurate beliefs, inaccurate beliefs increase (decrease) discrimination against group $F$ if, relative to type $\theta^{*}$ with the same preferences as $\theta$ and 
accurate beliefs, $\bar{s}(\theta, F) \geq(\leq) \bar{s}\left(\theta^{*}, F\right)$ and $\bar{s}(\theta, M) \leq(\geq) \bar{s}\left(\theta^{*}, M\right)$, with one inequality strict.

In other words, when inaccurate beliefs increase discrimination, type $\theta$ discriminates against group $F$ on a strictly larger set of signals than the type with the same preferences and accurate beliefs, whereas when inaccurate beliefs decrease discrimination, type $\theta$ discriminates against group $F$ on a strictly smaller set of signals. Our next result establishes that mistakenly assuming accurate beliefs leads to misidentified preferences. Further, depending on whether the inaccurate beliefs increase or decrease discrimination, the misidentified parameters will over- or underestimate preference partiality.

Observation 4 (Misidentified Preferences). Suppose a researcher incorrectly assumes an evaluator has accurate beliefs and uses the outcomes-based method to identify the evaluator's type.

1. For a generic set of types and true distributions, the researcher misidentifies preferences.

2. If inaccurate beliefs increase discrimination against group $F$, then the researcher overestimates the evaluator's preference partiality against group $F$, while if inaccurate beliefs decrease discrimination, then the researcher underestimates preference partiality.

In Fig. 3, if the evaluator believes that the average productivity for group $F$ is $\hat{\mu}_{F}=3$ when in fact it is $\mu_{F}=4.5$, then incorrectly imposing accurate beliefs will attribute discrimination stemming from this inaccurate belief to preference partiality, $u_{F}=6.3>u_{M}=6$, when in actuality, the evaluator has preference neutrality, $u_{F}=u_{M}=6$. In contrast, if the evaluator believes that the average productivity is the same for both groups, $\hat{\mu}_{F}=\hat{\mu}_{M}=5$ when in fact $\mu_{F}=4.5$, then imposing accurate beliefs will lead the researcher to underestimate the evaluator's preference partiality against group $F$, concluding $u_{F}=6.3$ when in fact it is equal to 6.4 .

Next, we establish one source of discrimination that the outcomes-based method can potentially rule out. Accurate statistical discrimination - that is, discrimination stemming from accurate belief partiality and preference neutrality - is of particular interest because it is often viewed as efficient from an informational perspective. In other words, if an evaluator is applying differential treatment to groups when underlying differences do exist, then this evaluator is simply engaging in profit-maximizing behavior. The outcomes-based method can rule out accurate statistical discrimination by showing that the observed pattern of discrimination is not consistent with accurate beliefs and preference neutrality. Ruling this out establishes that the discrimination either stems from animus towards a group or inaccurate beliefs about them. 
Observation 5 (Rejecting Accurate Statistical Discrimination). Suppose a researcher identifies the isodiscrimination curve with thresholds $\left(s_{M}, s_{F}\right)$ and observes $\left(\mu_{g}, \tau_{g}, \eta_{g}\right)$ for $g \in$ $\{M, F\}$. If $\frac{\tau_{M} \mu_{M}+\eta_{M} s_{M}}{\tau_{M}+\eta_{M}} \neq \frac{\tau_{F} \mu_{F}+\eta_{F} s_{F}}{\tau_{F}+\eta_{F}}$, the evaluator is not an accurate statistical discriminator.

In Fig. 3, the preferences and beliefs of an accurate statistical discriminator do not lie on the isodiscrimination curve, and therefore, are not consistent with the observed level of discrimination. Of course, when the observed pattern of discrimination is consistent with accurate statistical discrimination, this does not identify the evaluator as an accurate statistical discriminator: there are many other types that could also generate the observed behavior.

Importantly, even if a combination of preference partiality and inaccurate belief partiality is observationally equivalent to accurate statistical discrimination for the current hiring decision, inaccurate beliefs are not necessarily innocuous. These inaccurate beliefs may negatively affect the worker in future performance evaluations and promotions. For example, suppose an evaluator has beliefs that exaggerate the true differences in productivity between groups and preferences that somewhat favor the disadvantaged group through setting a lower hiring threshold $\left(u_{F}<u_{M}\right)$ for entry-level positions. And suppose this yields equivalent discrimination to the accurate statistical discrimination type. Then if the evaluator only feels compelled to favor the disadvantaged group for entry-level hiring, these inaccurate beliefs will lead to persistently lower rates of promotion and advancement.

Given the difficulty of using the outcomes-based method to identify the source of discrimination, we now explore two other possible methods: eliciting beliefs and manipulating information.

Eliciting Beliefs. If it is possible to collect data on the evaluator's subjective beliefs, then comparing hiring decisions to these beliefs can identify the source of discrimination.

Observation 6 (Identifying Preferences from Beliefs). Suppose a researcher can identify the isodiscrimination curve, i.e. from observing $v, g$, and $s$. Observing $\left(\hat{\mu}_{g}, \hat{\tau}_{g}, \hat{\eta}_{g}\right)$ identifies $u_{g}$, and therefore, the evaluator's type.

Importantly, observing subjective beliefs does not identify whether they are accurate - additional data, such as outcomes, is necessary to determine whether beliefs are accurate.

Manipulating Information. Another potential method to identify the source of discrimination is manipulating the amount of information presented to evaluators. We now demonstrate 
how this method can be used to partially identify the source of discrimination. For example, one could compare discrimination in a treatment in which only one customer review is revealed to a treatment in which five customer reviews are revealed. In the current set-up, suppose we can vary the number of draws of the signal that the evaluator observes for a worker. If an evaluator believes that a single draw of the signal has precision $\hat{\eta}_{g}$, then she believes that observing $x$ draws of this signal has precision $x \hat{\eta}_{g}$. The characterization of the optimal hiring rule and the isodiscrimination curves for $x$ draws are identical to the case of a single draw, substituting $x \hat{\eta}_{g}$ for $\hat{\eta}_{g}$.

Given an evaluator of type $\theta$, the following result characterizes the set of types that exhibit equivalent discrimination to $\theta$ across multiple informational treatments.

Proposition 3 (Equivalent Discrimination Across Informational Treatments). Suppose an evaluator of type $\theta=\left(u_{g}, \hat{\mu}_{g}, \hat{\tau}_{g}, \hat{\eta}_{g}\right)_{g \in\{M, F\}}$ observes either $x_{1} \geq 1$ or $x_{2} \neq x_{1}$ signal draws for each worker. Then the set of types

$$
\left\{\theta^{\prime}=\left(u_{g}, \hat{\mu}_{g}^{\prime}, \hat{\tau}_{g}^{\prime}, \hat{\eta}_{g}^{\prime}\right)_{g \in\{M, F\}} \mid \hat{\mu}_{g}^{\prime}=u_{g}-\frac{\hat{\tau}_{g} / \hat{\eta}_{g}}{\hat{\tau}_{g}^{\prime} / \hat{\eta}_{g}^{\prime}}\left(u_{g}-\hat{\mu}_{g}\right)\right\}
$$

exhibit equivalent discrimination to $\theta$ across both informational treatments. This set of types also exhibit equivalent discrimination for any number $x \geq 1$ of signal draws.

This result establishes that there is a unique level of preference partiality that yields equivalent discrimination across multiple informational treatments. Therefore, manipulating the number of signal draws can identify the level of preference partiality. However, it is not possible to identify the form of belief partiality: given the identified preference parameter $u_{g}$ for each group and a type with beliefs $\left(\hat{\mu}_{g}, \hat{\tau}_{g}, \hat{\eta}_{g}\right)$, any type with beliefs $\left(\hat{\mu}_{g}^{\prime}, \hat{\tau}_{g}^{\prime}, \hat{\eta}_{g}^{\prime}\right)$ such that $\mu_{g}^{\prime}=u_{g}-\frac{\hat{\tau}_{g} / \hat{\eta}_{g}}{\hat{\tau}_{g}^{\prime} / \hat{\eta}_{g}^{\prime}}\left(u_{g}-\hat{\mu}_{g}\right)$ for each group will exhibit equivalent discrimination. The following observation summarizes this insight.

Observation 7 (Identifying Preferences from Manipulating Information). Suppose a researcher identifies the isodiscrimination curves for at least two informational treatments. This identifies the evaluator's preferences $u_{g}$, but does not identify the evaluator's beliefs $\left(\hat{\mu}_{g}, \hat{\tau}_{g}, \hat{\eta}_{g}\right)$.

Fig. 4 shows how it is possible to identify a unique preference parameter by varying the number of signal draws. Only a type with preference parameter $u_{F}=6.2$ exhibits the observed discrimination for both informational treatments. The other types on the isodiscrimination curve for one draw exhibit equivalent discrimination to the type with $u_{F}=6.2$ 


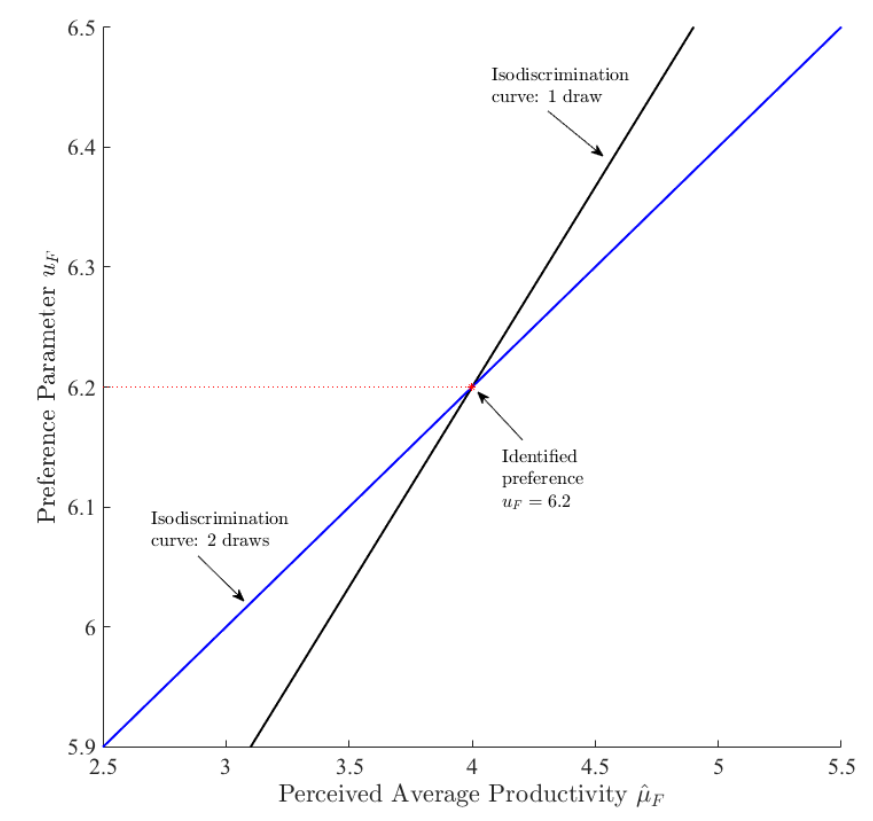

Figure 4. Information Manipulation

Isodiscrimination curves for $\left(u_{F}, \hat{\mu}_{F}, \hat{\tau}_{F}, \hat{\eta}_{F}\right)=(6.2,4, .5,1)$

when there is a single draw. But these types do not exhibit equivalent discrimination to the type with $u_{F}=6.2$ when there are two signal draws - as these types do not lie on the isodiscrimination curve for two draws. While it may look like the perceived average productivity $\hat{\mu}_{F}=4$ is also identified, this is just the belief for a type $\theta$ with perceived concentration $\hat{\tau}_{F}=.5$ and signal precision $\hat{\eta}_{F}=1$. There are other types with different values of $\left(\hat{\tau}_{F}, \hat{\eta}_{F}\right)$ that exhibit equivalent discrimination across all informational treatments, and these types will have different values of $\hat{\mu}_{F}$ (but the same preference $u_{F}=6.2$, of course, since the preference parameter is identified). For example, from Proposition 3, a type $\theta^{\prime}$ with $u_{F}=6.2$, $\tau_{F}^{\prime}=.4, \eta_{F}^{\prime}=1$ and $\hat{\mu}_{F}^{\prime}=6.2-\frac{.5 / 1}{4 / 1}(6.2-4)=3.45$ will exhibit equivalent discrimination to $\theta$ for any number of signal draws.

A crucial feature of this information manipulation is that the multiple signals are drawn from the same distribution. In other words, for each group, the evaluator has the same model of the precision for each draw of the signal. This contrasts with an information manipulation in which additional signals from other domains are included - for example, comparing discrimination in a treatment in which education is revealed to a treatment in which education and SAT score are revealed. In this case, the evaluator may have a different group-specific model of the signal distribution for each type of signal - for example, the evaluator believes 
that education is more informative for group $M$, while SAT score is more informative for group $F$. It is not possible to identify the evaluator's preference partiality - or any other aspect of her type - from varying the set of observed signals across different domains.

Taken together, either the belief elicitation or information manipulation method can separate preference partiality from belief partiality. If it is possible to elicit an evaluator's beliefs for all parameters of the relevant distributions, then it is possible to fully identify the evaluator's type. In practice, this will be difficult in certain settings - both due to the complexity and reliability of methods for eliciting beliefs about higher moments and due to the feasibility of collecting such information (for example, it may not be possible to collect beliefs in certain settings such as on an online platform). Therefore, the information manipulation method provides an alternative, simpler way to identify preferences and "aggregate" belief partiality - although it comes at the cost of not being able to separate the different ways that beliefs can be inaccurate.

\section{Identifying the Source}

In this section, we employ a stylized experimental setting to demonstrate the pitfalls of the identification problem posed by inaccurate beliefs - in particular, how assuming accurate beliefs can lead to erroneous conclusions - and propose a potential methodology to solve it. The experiment allows us to perform the accounting exercise employed in outcomes-based tests and to also elicit beliefs about relevant characteristics. We show that average beliefs are incorrect, violating the accurate beliefs assumption typically made when considering statistical discrimination, and that ignoring these inaccurate beliefs leads to a false identification of the source of discrimination. We also demonstrate how informational interventions can be used both to separate inaccurate beliefs from underlying animus and to correct these inaccurate beliefs. Specifically, we inform individuals of the true group-specific average productivities. Participants adjust their behavior significantly in the direction of the information, suggesting that at least some of the observed discrimination is driven by inaccurate beliefs rather than animus.

\subsection{Experimental Design}

In this section, we provide a short summary of the pre-registered experimental design; we outline the design in detail in Appendix B. We recruited two samples of subjects on Amazon Mechanical Turk (participants) to complete either a work task (Survey 1) or a hiring task (Survey 2). In the first survey, we recruited 589 participants to create a population of 
"workers" (392 from the US and 197 from India). These participants answered a set of demographic questions, followed by 50 multiple-choice math questions. They were told that their performance would not affect their payment. This design provided for relatively continuous and precise measures of productivity, and allowed us to study discrimination without using deception.

In the second survey, we recruited 577 different participants to create a population of "employers" (392 from the US, 185 from India). These participants were told about the task assigned to "workers" (they were shown five examples of the questions), that the average score was 36.95 out of 50, and that they would serve as an "employer" who could potentially hire one of the workers. The hiring task involved multiple stages. In the first stage, each employer was shown 20 profiles of potential workers, randomly selected from the bank, and made a wage offer for each worker. The profiles included demographic variables which could be informative for forming beliefs about productivity (age, gender, and nationality), or over which employers may hold taste-based motives, as well as potentially irrelevant information (favorite high school subject, sport, color, movie, and coffee/tea preference); see Fig. B1 for an example. One of these workers was selected and their wage offer was accepted or rejected according to the Becker-DeGroot-Marschak mechanism to incentivize truthful reporting. Specifically, if the wage offer was larger than a randomly generated number, then it was 'accepted' - the employer paid the random number as a bonus to the selected worker and the employer received 1 cent for each question answered correctly (in addition to a $\$ 2$ participation payment); if the wage offer was smaller than the random number, it was 'rejected' - the employer paid nothing and the worker did not receive a bonus. ${ }^{9}$

In the second stage, we elicited employers' beliefs about the average productivity of different groups (men/women, residents from the US/India, people above/below the median age of 33), randomizing whether or not this was incentivized. ${ }^{10}$ The third stage involved the informational intervention, which provided employers with average productivity data for each of the six groups. The fourth stage involved a second set of hiring decisions for 10 worker profiles, which was conducted in the same way as the first stage.

\footnotetext{
${ }^{9}$ Employers saw examples of the mechanism and passed comprehension checks before making wage offers.

${ }^{10} \mathrm{We}$ only elicited beliefs about the first moment of the performance distribution. While participants may also have inaccurate beliefs about other statistics, demonstrating a difference in perceived versus actual means is sufficient to falsify the assumption that beliefs are correct, which was the primary goal of the illustrative experiment. Eliciting other moments of the distribution, e.g. variance, is more complex for participants relative to eliciting the mean. Given the multiple stages in the study, we sought to keep the belief elicitation task as simple as possible in order to curtail potential confusion and minimize noise.
} 


\subsection{Experimental Results}

A necessary prerequisite to study the source of discrimination is to find a context and a population in which discrimination occurs. Ex-ante, it was not obvious that our stylized hiring experiment would satisfy this requirement. The employers knew that they were being observed as part of a research study and the relevant group information was represented abstractly (e.g. written text) rather than viscerally (e.g. a picture). All of these factors may attenuate the influence of animus. ${ }^{11}$

Despite these attenuating factors, we did find evidence of discrimination with respect to two out of three group identities: gender and nationality. Panel A of Table 4 presents the differences in average wages paid by employers to worker profiles from each group. With respect to gender, male profiles were paid on average 31.90 cents, while female profiles were paid 30.85 cents, a significant $3.4 \%$ difference $(p<0.01)$. With respect to nationality, profiles from India were favored, earning an average of 32.85 cents, while profiles from the U.S. earned 30.71 cents, a significant $7.0 \%$ difference $(p<0.01)$. Finally, there was modest evidence of age discrimination: subjects at or below age 33 were paid an average of 31.67 cents and those above age 33 were paid 31.14 cents, a $1.7 \%$ difference that is significant at the $5 \%$ level $(p=0.02)$. To put these differences into context, we show a "placebo" comparison for a profile characteristic that was unlikely to be either a target of taste-based motives or a proxy for math ability: the worker's preference for tea versus coffee. We find a similar level of discrimination to the level that we found for age, with tea drinkers earning a $1.7 \%$ higher average wage (31.74 vs. 31.22 cents), significant at the $5 \%$ level $(p=0.03)$. Table C3 demonstrates that these results are robust to demographic controls and employer fixed effects.

To examine the possibility of in-group bias, we run similar regressions controlling for the employer belonging to the group of interest (e.g. female) and the interaction of the two indicators to measure in-group bias (see Table C4). We find that the interaction is insignificant for gender and marginally significant for nationality, although in the direction of favoring the out-group. For age, we find a significant interaction effect. This suggests that the null effect in Table 4 masks in-group bias by both older and younger employers. Antonovics and Knight (2009) use a similar set of regressions to test for taste-based discrimination. This specification is motivated by the assumption that animus varies between groups (i.e. there is less animus toward one's in-group than out-group), but that beliefs are similar across groups (since they are taking a "standard model of statistical discrimination" as the benchmark and

\footnotetext{
${ }^{11}$ For example, Bar and Zussman (2019) argue that a lack of interaction may attenuate the extent of taste-based discrimination in driving test examinations.
} 
Table 4. Wages and "Productivities", by Employee Characteristics (Hiring Task 1)

\begin{tabular}{|c|c|c|c|c|c|c|}
\hline & $\begin{array}{c}\text { Group } 1 \\
(1)\end{array}$ & $\begin{array}{c}\text { Group } 2 \\
(2)\end{array}$ & $\begin{array}{c}\text { Diff. } \\
(3)\end{array}$ & $\begin{array}{c}\text { p-val } \\
(4)\end{array}$ & $\underset{(5)}{\# \text { Obs. G1 }}$ & $\begin{array}{c}\# \text { Obs. G2 } \\
(6)\end{array}$ \\
\hline \multicolumn{7}{|c|}{ Panel A: Employers' Wage WTP, by Employee Characteristics } \\
\hline Gender ( $1=$ Male, $2=$ Female $)$ & $\begin{array}{c}31.90 \\
(12.07)\end{array}$ & $\begin{array}{c}30.85 \\
(12.23)\end{array}$ & 1.05 & 0.00 & 6,306 & 5,234 \\
\hline Country $(1=\mathrm{US}, 2=$ India $)$ & $\begin{array}{c}30.71 \\
(12.20)\end{array}$ & $\begin{array}{c}32.85 \\
(11.95)\end{array}$ & -2.14 & 0.00 & 7,700 & 3,840 \\
\hline Age $(1=$ Under $33,2=$ Over 33$)$ & $\begin{array}{c}31.67 \\
(12.00)\end{array}$ & $\begin{array}{c}31.14 \\
(12.33)\end{array}$ & 0.54 & 0.02 & 6,139 & 5,401 \\
\hline Placebo ( $1=$ Prefer Coffee, $2=$ Prefer Tea $)$ & $\begin{array}{c}31.22 \\
(12.32)\end{array}$ & $\begin{array}{c}31.74 \\
(11.89)\end{array}$ & -0.52 & 0.03 & 7,075 & 4,465 \\
\hline \multicolumn{7}{|c|}{ Panel B: Employee Productivity, by Employee Characteristics } \\
\hline Gender $(1=$ Male, $2=$ Female $)$ & $\begin{array}{l}38.30 \\
(8.55)\end{array}$ & $\begin{array}{l}34.98 \\
(8.73)\end{array}$ & 3.32 & 0.00 & 6,306 & 5,234 \\
\hline Country $(1=\mathrm{US}, 2=$ India $)$ & $\begin{array}{l}37.01 \\
(8.93)\end{array}$ & $\begin{array}{l}36.36 \\
(8.49)\end{array}$ & 0.65 & 0.00 & 7,700 & 3,840 \\
\hline Age $(1=$ Under $33,2=$ Over 33$)$ & $\begin{array}{l}36.96 \\
(8.62)\end{array}$ & $\begin{array}{l}36.60 \\
(8.98)\end{array}$ & 0.37 & 0.03 & 6,139 & 5,401 \\
\hline Placebo ( $1=$ Prefer Coffee, $2=$ Prefer Tea) & $\begin{array}{l}36.64 \\
(8.77)\end{array}$ & $\begin{array}{l}37.03 \\
(8.82)\end{array}$ & -0.40 & 0.02 & 7,075 & 4,465 \\
\hline
\end{tabular}

Notes: Standard deviations in parentheses. One observation per worker-employer combination. Column (4) shows the p-value from two-sample t-tests for the equality of columns (1) and (2).

note that "these beliefs must be correct in equilibrium"). In Table C5, we test this assumption in our experimental environment. We find that beliefs about the gender performance gap are identical among both female and male employers. However, for nationality, we find a significant difference in beliefs, namely, Indians hold beliefs that more strongly favor the out-group (Americans).

Having demonstrated moderate levels of discrimination in hiring, we now examine the "ground truth" in actual productivity differences between groups. The typical outcomesbased test of statistical discrimination requires mapping disparities between groups in the evaluators' relevant decision (e.g. the wages offered to employees) to disparities in an outcome in the evaluators' objective function (e.g. the employees' productivity). ${ }^{12}$ In our context, this requires mapping disparities in the employers' to stated wages to disparities in group-specific productivity differences, i.e. the number of questions answered correctly. The commonly used outcome method compares disparities in wages to disparities in performance to measure the relative role of (accurate) statistical versus taste-based discrimination (in the context of our

\footnotetext{
${ }^{12}$ Translating the two measures may require strong modeling assumptions (e.g. whether there is heterogeneity in the search costs faced by evaluators). For discussions of these assumptions in the context of the hit-rate tests, see Antonovics and Knight (2009); Dharmapala and Ross (2004); Anwar and Fang (2006).
} 
framework, accurate belief-based versus preference-based partiality). For simplicity, we will refer to both disparities as measured in "points."

Panel B of Table 4 shows the average number of correct answers by each sub-group (see Fig. C2 for probability density functions). As shown in Panel A of Table 4, the gap in average wages for men and women was lower than the gap in average performance (1.05 points versus 3.32 points). ${ }^{13}$ Therefore, if we used the standard outcome method to separate statistical and taste-based discrimination, we would conclude that the entire 1.05 point disparity in wages is due to (accurate) statistical discrimination - the remaining 2.27 point difference in performance would be attributed to taste-based prejudice against men. Turning to nationality-based discrimination, there was a wage gap of -2.14 points in favor of Indians, compared to a performance gap of 0.65 points in favor of Americans. Under the standard approach, we would conclude that both the -2.14 point disparity in wages, when compared to the +0.65 point difference in performance, suggests taste-based prejudice against Americans. $^{14}$

We now proceed to examine whether inaccurate beliefs can explain the disparities in compensation. As an initial check to see whether employers' decisions were guided by the elicited beliefs, we correlate wages with their beliefs about group-specific productivities. We find positive correlations for all six groups of workers (Female: 0.12, Male: 0.12, India: 0.15, U.S.: 0.12, Over 33: 0.12, Under 33: 0.10). Given that we elicited beliefs after the hiring task, it is possible that part of these correlations are due to rationalization (e.g. an individual first discriminates against women when setting wages, then chooses beliefs to justify this decision), or audience effects (e.g. an individual falsely reports beliefs that justify the discriminatory decision to the experimenter). To test for this, we provided half of the employees with large incentives for belief accuracy. In Table C2, we show that beliefs are nearly identical across both incentive conditions, with none of the six comparisons being significantly different from one another. Together these findings suggest that the employers' group-specific performance

\footnotetext{
${ }^{13}$ We calculate productivity differences using the full sample of profiles observed in hiring task 1 . This is a weighted sample of the original population of 577 workers (since each of the 589 employers saw independent random samples of 20 of the 577 workers). Due to the random variation in the profiles observed, the grouplevel averages slightly differ from those found in Table B1. For example, the male-female performance gap is 3.04 points in Table B1 and 3.32 points in this weighted sample. Note that the averages in Table B1 are the basis for the informational intervention.

${ }^{14}$ While we document significant discrimination by gender (i.e. men are paid more than women), the outcome method reveals that the performance gap exceeds the pay gap. This leads to the conclusion that there is taste-based discrimination against men. While the literature often equates taste-based discrimination with animus or prejudice, this link is inappropriate when discrimination manifests as an equalizing action. For example, people may be equalizing wages between two groups despite differences in productivity due to fairness concerns. We discuss the implications of this distinction further in the conclusion.
} 
Table 5. Beliefs about Productivity by Employee Characteristics

\begin{tabular}{lcccc}
\hline & $\begin{array}{c}\text { Group 1 } \\
(1)\end{array}$ & $\begin{array}{c}\text { Group 2 } \\
(2)\end{array}$ & $\begin{array}{c}\text { Diff. } \\
(3)\end{array}$ & $\begin{array}{c}\text { p-val } \\
(4)\end{array}$ \\
\hline Gender (1=Male, 2=Female) & 34.04 & 32.14 & 1.89 & 0.00 \\
& $(8.26)$ & $(8.41)$ & & \\
Country (1=US, 2=India) & 32.08 & 34.80 & -2.72 & 0.00 \\
& $(8.56)$ & $(9.44)$ & & \\
Age (1=Under 33, 2=Over 33) & 33.41 & 31.57 & 1.84 & 0.00 \\
& $(8.97)$ & $(9.00)$ & & \\
\hline
\end{tabular}

Notes: Standard deviations in parentheses. One observation per employer combination. Column (4) shows the p-value from one-sample t-tests for the equality of columns (1) and (2). \# Observations $=577$.

predictions provide meaningful information about their true beliefs.

In Table 5, we present employer beliefs about the group-specific average performance, which can be compared directly to the actual group-specific performance reported in Table 4, Panel B. Predictions about performance are lower than actual performance for all six groups. This overall underestimation is consistent with risk aversion (recall that employers face the potential of a negative payment, taken from their $\$ 0.50$ bonus, if they overestimate performance). Consistent with this, gaps in beliefs about performance are larger than gaps in wage payments. Using employers' actual beliefs to identify the source of discrimination leads to substantially different conclusions than the outcomes-based method outlined above. Looking at nationality, the wage gap is -2.14 points and the performance gap is +0.65 points; the gap in beliefs is -2.72 points. Thus, the entire wage gap can be explained by inaccurate beliefs. In contrast to the outcome method which infers taste-based discrimination in favor of Indian workers, the remaining 0.58 point difference between the belief and wage gaps suggests prejudice against them. Looking at gender, the wage gap is 1.05 points, the performance gap is 3.32 points, and the belief gap is 1.89 points. The majority of the wage gap can be explained by inaccurate beliefs: the residual attributed to preference-based sources shrinks from 2.17 to 0.84 points. Finally, despite the minimal gap in wages and performance based on age, employers believed that young workers will significantly outperform older ones. This suggests some preference-based partiality against younger workers. Together these results highlight that a failure to account for inaccurate statistical discrimination may lead to the wrong conclusion on the source of treatment disparities.

To identify whether the observed disparate treatment was driven by inaccurate statistical discrimination or animus-driven beliefs, we examined how behavior would respond to an informational intervention. Table 6 compares the differences between the two hiring rounds 
Table 6. Effect of Information: Difference-in-Differences by Hiring Task

\begin{tabular}{lcccccc}
\hline & $(1)$ & $(2)$ & $(3)$ & $(4)$ & $(5)$ & $(6)$ \\
\hline Post-Info & $1.53^{* * *}$ & $1.60^{* * *}$ & $1.06^{* * *}$ & $1.28^{* * *}$ & $1.94^{* * *}$ & $2.39^{* * *}$ \\
& $(0.29)$ & $(0.27)$ & $(0.31)$ & $(0.29)$ & $(0.44)$ & $(0.36)$ \\
Female & $-1.05^{* * *}$ & & & & $-0.67^{* * *}$ & $-0.81^{* * *}$ \\
& $(0.25)$ & & & & $(0.24)$ & $(0.19)$ \\
Female*Post-Info & $-0.64^{*}$ & & & & $-0.90^{* *}$ & $-1.00^{* * *}$ \\
& $(0.37)$ & & & & $(0.37)$ & $(0.29)$ \\
Indian & & $2.14^{* * *}$ & & & $1.98^{* * *}$ & $1.99^{* * *}$ \\
& & $(0.29)$ & & & $(0.29)$ & $(0.25)$ \\
Indian*Post-Info & & $-1.07^{* * *}$ & & & $-1.20^{* * *}$ & $-1.63^{* * *}$ \\
& & $(0.40)$ & & & $(0.42)$ & $(0.34)$ \\
Over 33 & & & $-0.54^{* *}$ & & 0.07 & 0.30 \\
& & & $(0.26)$ & & $(0.26)$ & $(0.22)$ \\
Over 33*Post-Info & & & 0.41 & & 0.14 & -0.21 \\
& & & $(0.40)$ & & $(0.42)$ & $(0.30)$ \\
Prefers Tea & & & & $0.52^{* *}$ & $0.39^{*}$ & $0.35^{* *}$ \\
& & & & $(0.24)$ & $(0.24)$ & $(0.18)$ \\
Prefers Tea*Post-Info & & & & -0.08 & 0.06 & -0.18 \\
& & & & $(0.38)$ & $(0.38)$ & $(0.27)$ \\
\hline \# Observations & 17,310 & 17,310 & 17,310 & 17,310 & 17,310 & 17,310 \\
$R^{2}$ & 0.01 & 0.01 & 0.00 & 0.00 & 0.01 & 0.48 \\
DepVarMean & 31.90 & 30.71 & 31.67 & 31.22 & 30.18 & 30.18 \\
Employer FE? & No & No & No & No & No & Yes \\
\hline$*$
\end{tabular}

${ }^{*} p<0.10,{ }^{* *} p<0.05,{ }^{* * *} p<0.01$

Notes: Standard errors in parentheses, clustered by employer. "DepVarMean" is the mean of the dependent variable (wage WTP) in the omitted group (e.g. Male Workers in Hiring Task 1 for column (1)). "Post-Info" is an indicator for whether a profile came in the second hiring task (i.e. profiles 21-30 of the 30 total profiles evaluated). The observed performance (trivia score) averages for the sample of profiles observed in Hiring Task 2 are: 38.13 (Male), 35.13 (Female), 36.95 (US), 36.53 (India), 36.84 (Under 33), 36.77 (Over 33), 36.81 (Prefer Coffee), 36.79 (Prefer Tea).

("Post-Info"), the differences between wages assigned to profiles of each demographic group (e.g. "Female"), and the difference-in-differences (e.g. "Female X Post-Info"). The coefficients on "Post-Info" suggests substantial belief updating across all demographic groups, partially correcting the large level differences in the first hiring task between wages and actual groupspecific productivity (a gap of roughly 5 points on average). The effect of the informational intervention on hiring decisions suggests that the majority of initial discrimination was driven by inaccurate beliefs rather than accurate statistical or preference-based sources. ${ }^{15}$

\footnotetext{
${ }^{15}$ There are several caveats to note when interpreting these results. Beliefs were not measured a second time. Additionally, experimenter demand may have played a role, though recent work suggests that this factor is likely small (De Quidt, Haushofer, and Roth, 2018). Finally, the change in wages could reflect an
} 


\section{Conclusion}

The study of discrimination and its motives has a rich history in economics. Separating out statistical and taste-based drivers of discrimination is a useful exercise, but as our survey of the literature illustrates, it has thus far relied heavily on the assumption of accurate beliefs. There are many reasons to suspect that beliefs may not always be accurate. This paper formally outlines the identification problem inherent in distinguishing between belief-based and preference-based motives. A stylized experiment is used to highlight the pitfalls of not accounting for inaccurate beliefs when attempting to identify the source of discrimination, and illustrates a potential methodology for improved identification.

The results of the information intervention suggest that identifying inaccurate beliefs may have immediate policy implications for reducing discrimination. However, there are some important caveats to keep in mind when considering how this type of intervention would be implemented outside of the stylized exercise. First, such an intervention is likely feasible only in contexts where the underlying target outcome (e.g. productivity) is reliably measured and reflects the appropriate counterfactual outcome for all groups. To the first point, the accuracy of the underlying outcomes may differ by group; for example, police officers have been shown to be more likely to discount the recorded speed of a white driver than a minority driver (Goncalves and Mello, 2019). To the latter point, there are contexts in which discrimination at (often unobserved) intermediate stages renders final productivity measures unreliable due to behavioral responses. For example, minority pitchers correctly anticipate discrimination by umpires and modify their behavior, resulting in a downward bias for performance measures (Parsons, Sulaeman, Yates, and Hamermesh, 2011). Studies have also documented that bias at intermediate stages can skew final productivity measures among grocery store workers (Glover, Pallais, and Pariente, 2017) and academic economists (Hengel, 2019). It is important to also take into account the underlying psychology of how people will respond to the information. Selection decisions such as hiring are rarely onedimensional. Drawing attention to a (smaller than expected) productivity gap could correct beliefs, while nonetheless increasing discrimination if it increases the salience of the gap as an input into the hiring decision. These concerns highlight the need for future tests that operationalize and examine similar informational interventions in field contexts.

experience effect between assigning wages in the first and second hiring task. To investigate this channel, we perform a test comparing the average wages assigned in the first 10 profiles and the second 10 profiles during the initial task. We do not find evidence for an experience effect (36.86 vs. 36.72; $\mathrm{p}=.39)$. While we cannot fully rule out all these possible confounds, we view the information intervention as a proof of concept for the type of methodology that can be used as both an intervention for correcting beliefs and methodology identifying belief-based discrimination from preference-based motives. 
Throughout the paper, we document discrimination in wages by gender (i.e. men paid more than women). Carrying out the standard outcomes-based method reveals that the gap in performance exceeds that of the gap in pay. This leads to the conclusion that there is preference-based partiality against the group that received higher wages - male workers. While taste-based discrimination is often used as a synonym for animus or prejudice against a group, this link seems misplaced when discrimination manifests as an equalizing actions (e.g. equalizing wage rates). For example, people may treat groups similarly regardless of actual or believed productivity differences due to fairness concerns. Additionally, there is often an equity-efficiency trade-off to discrimination, such that even in the absence of legal or social sanctions, an employer may wish to equalize wages across groups (for a theoretical discussion of these trade-offs in the context of racial profiling, see Durlauf (2005)). Such a concern may be especially pronounced for wages, where even abstracting away from group-level attributes, there is evidence that fairness norms may contribute to observed wage compression (e.g. Breza, Kaur, and Shamdasani (2018))

Just as decomposing the nature of belief-based discrimination has implications for policy, the same may be true for preference-based partiality. For example, if the basis for preferencebased partiality is animus or prejudice, then a policy that increases contact between groups may reduce disparities (Dobbie and Fryer, 2015; Paluck, Green, and Green, 2018). By contrast, if the behavior is instead sanction- or value-oriented, then such interventions will likely have little impact. While it is difficult to imagine a simple elicitation that would allow for a parsimonious quantitative decomposition of "tastes", survey measures may be able to make some headway in this endeavor. Such a decomposition is outside of the scope of this paper, but future work along these lines would enrich our understanding of discrimination, and help in the development of tools used to identify it and design policy.

Lastly, our findings speaks to the need for continued work such as Bordalo et al. (2016) that may help to identify situations when inaccurate beliefs are especially likely to be prevalent. As research begins to identify situations where inaccurate beliefs are a driving factor for discrimination, future work will hopefully also begin to develop policy interventions that are able to effectively correct beliefs and reduce discrimination as a result.

\section{References}

Agan, A. And S. Starr (2017): "Ban the Box, Criminal Records, and Racial Discrimination: A Field Experiment," The Quarterly Journal of Economics, 133, 191-235.

Aigner, D. J. And G. G. Cain (1977): "Statistical Theories of Discrimination in Labor 
Markets," ILR Review, 30, 175-187.

Albrecht, K., E. Von Essen, J. Parys, And N. Szech (2013): "Updating, selfconfidence, and discrimination," European Economic Review, 60, 144-169.

Antonovics, K. And B. G. Knight (2009): "A New Look at Racial Profiling: Evidence from the Boston Police Department," Review of Economics and Statistics, 91, 163-177.

AnWAR, S. AND H. FAng (2006): "An alternative test of racial prejudice in motor vehicle searches: Theory and evidence," American Economic Review, 96, 127-151.

Arnold, D., W. DobBie, And C. YAng (2018): "Racial Bias in Bail Decisions," Quarterly Journal of Economics, 1885-1932.

Arrow, K. J. (1973): "The Theory of Discrimination," in Discrimination in Labor Markets, ed. by O. Ashenfelter and A. Rees, Princeton, NJ: Princeton University Press.

Ayres, I. (2002): "Outcome Tests of Racial Disparities in Police Practices," Justice Research and Policy, 4, 131-142.

BAR, R. And A. Zussman (2019): "Identity and Bias: Insights from Driving Tests," Working Paper, 1-45.

Bartoš, V., M. Bauer, J. Chytilová, and F. MatěJka (2016): "Attention Discrimination: Theory and Field Experiments with Monitoring Information Acquisition," American Economic Review, 106, 1437-75.

Beaman, L., R. Chattopadhyay, E. Duflo, R. Pande, and P. Topalova (2009): "Powerful Women: Does Exposure Reduce Bias?" The Quarterly Journal of Economics, $124,1497-1540$.

Becker, G. (1957): The Economics of Discrimination, Chicago: University of Chicago Press.

Bertrand, M. And E. Duflo (2017): "Field Experiments on Discrimination," Handbook of Field Experiments, 1, 110.

Bertrand, M. And S. Mullainathan (2004): "Are Emily and Greg More Employable Than Lakisha and Jamal? A Field Experiment on Labor Market Discrimination," American Economic Review, 94, 991-1013. 
Bohren, J. A., A. Imas, and M. Rosenberg (2019): "The Dynamics of Discrimination: Theory and Evidence," Working Paper.

Bordalo, P., K. Coffman, N. Gennaioli, And A. Shleifer (2016): "Stereotypes," Quarterly Journal of Economics, 1753-1794.

Brenm, J. W. (1966): A Theory of Psychological Reactance, New York: Academic Press.

Breza, E., S. Kaur, And Y. Shamdasani (2018): "The morale effects of pay inequality," Quarterly Journal of Economics, 133, 611-663.

Bursztyn, L., A. L. González, And D. Yanagizawa-Drott (2018): "Misperceived social norms: Female labor force participation in Saudi Arabia," Tech. rep., National Bureau of Economic Research.

Cameron, S. V. and J. J. Heckman (2001): "The Dynamics of Educational Attainment for Black, Hispanic, and White Males," Journal of Political Economy, 109, 455-499.

Charles, K. K. and J. Guryan (2011): "Studying Discrimination: Fundamental Challenges and Recent Progress," Annual Review of Economics, 3, 479-511.

De Quidt, J., J. Haushofer, and C. Roth (2018): "Measuring and bounding experimenter demand," American Economic Review, 108, 3266-3302.

Dharmapala, D. And S. L. Ross (2004): "Racial bias in motor vehicle searches: Additional theory and evidence," Contributions to Economic Analysis and Policy, 3, 89-111.

Dobbie, W. And R. G. Fryer (2015): "The Impact of Youth Service on Future Outcomes: Evidence from Teach for America," The B.E. Journal of Economic Analysis and Policy, 15, 1031-1066.

Durlauf, S. N. (2005): "Racial Profiling as a Public Policy Question: Efficiency, Equity, and Ambiguity," American Economic Review, 95, 132-136.

FAnG, H. AND A. Moro (2011): "Theories of statistical discrimination and affirmative action: A survey," in Handbook of social economics, Elsevier, vol. 1, 133-200.

Fershtman, C. And U. Gneezy (2001): "Discrimination in a Segmented Society: An Experimental Approach," Quarterly Journal of Economics, February, 351-377. 
Fiske, S. T. (1998): "Stereotyping, prejudice, and discrimination," The handbook of social psychology, 2, 357-411.

Gagnon-Bartsch, T., M. Rabin, and J. Schwartzstein (2018): Channeled attention and stable errors, Harvard Business School.

Glover, D., A. Pallais, And W. Pariente (2017): "Discrimination as a Self-Fulfilling Prophecy: Evidence from French Grocery Stores," Quarterly Journal of Economics, 12191260.

Gneezy, U., M. Niederle, And A. Rustichini (2003): "Performance in competitive Environments: Gender differences," Quarterly Journal of Economics, 1049-1074.

Goncalves, F. And S. Mello (2019): "A Few Bad Apples? Racial Bias in Policing," Working Paper, 1-79.

Haavelmo, T. (1944): "The Probability Approach in Econometrics," Supplement to Econometrica, 12, 1-115.

Hedegaard, M. S. And J.-R. Tyran (2018): "The Price of Prejudice," American Economic Journal: Applied Economics, 10, 40-63.

Hengel, E. (2019): "Publishing while female," Working Paper, 1-67.

Hilton, J. L. And W. V. Hippel (1996): "Stereotypes," Annual Review of Psychology, 47, $237-271$.

HÜBert, R. And A. T. Little (2020): "A Behavioral Theory of Discrimination in Policing," Working Paper.

JENSEN, R. (2010): "The (perceived) returns to education and the demand for schooling," Quarterly Journal of Economics, 515-548.

JudD, C. M. AND B. PARK (1993): "Definition and assessment of accuracy in social stereotypes," Psychological Review, 100, 109-128.

Knowles, J., N. Persico, And P. Todd (2001): "Racial bias in motor vehicle searches: Theory and evidence," Journal of Political Economy, 109, 203-229.

Knox, D. E., W. Lowe, And J. Mummolo (2020): "Administrative Records Mask Racially Biased Policing," American Political Science Review, 1-19. 
Kravitz, D. A. And J. Platania (1993): "Attitudes and Beliefs About Affirmative Action: Effects of Target and of Respondent Sex and Ethnicity," Journal of Applied Psychology, 78, 928-938.

LePage, L.-P. (2020): "Endogenous Learning and the Persistence of Employer Biases in the Labor Market," Tech. rep., mimeo.

List, J. A. (2004): "The Nature and Extent of Discrimination in the Marketplace: Evidence from the Field," Quarterly Journal of Economics, 49-89.

Mobius, M. And T. Rosenblat (2006): "Why Beauty Matters," American Economic Review, 96, 222-235.

Norton, M. I. And D. Ariely (2011): "Building a better America-one wealth quintile at a time," Perspectives on Psychological Science, 6, 9-12.

Paluck, E. L., S. Green, And D. P. Green (2018): "The Contact Hypothesis Reevaluated," Behvioural Public Policy, 1-30.

Parsons, C. A., J. Sulaeman, M. C. Yates, and D. S. Hamermesh (2011): "Strike Three: Discrimination, Incentives, and Evaluation," American Economic Review, 101, $1410-1435$.

Phelps, E. S. (1972): "The Statistical Theory of Racism and Sexism," American Economic Review, 62, 659-661.

Pope, D. G. And J. R. Sydnor (2011): "What's in a Picture? Evidence of Discrimination from Prosper.com," The Journal of Human Resources, 46, 53-92.

Schneider, D., A. Hastorf, And P. Ellsworth (1979): Person Perception, Reading, MA: Addison-Wesley.

Simoiu, C., S. Corbett-Davies, And S. Goel (2017): "The problem of infra-marginality in outcome tests for discrimination," Annals of Applied Statistics, 11, 1193-1216.

Zimmermann, F. (2019): "The Dynamics of Motivated Beliefs," Working Paper. 


\section{Appendix A. Proofs from Section 3}

Proof of Lemma 1. The normal distribution is the conjugate prior for a normal likelihood function. Therefore, the evaluator's posterior belief about productivity is normally distributed,

$$
a \mid s \sim N\left(\frac{\hat{\tau}_{g} \hat{\mu}_{g}+\hat{\eta}_{g} s}{\hat{\tau}_{g}+\hat{\eta}_{g}}, \frac{1}{\hat{\tau}_{g}+\hat{\eta}_{g}}\right) .
$$

This implies the optimal decision rule is $v(s, g, \theta)=1$ iff $\frac{\hat{\tau}_{g} \hat{\mu}_{g}+\hat{\eta}_{g} s}{\hat{\tau}_{g}+\hat{\eta}_{g}} \geq u_{g}$. Rearranging terms to represent this decision rule as a cut-off with respect to the signal results in Eq. (1).

Proof of Proposition 1. This follows from Eq. (1) and the discussion in the text. If two types do not discriminate, which corresponds to setting the same thresholds for each group, then trivially they exhibit equivalent discrimination, even when they set different signal thresholds from each other.

Proof of Observation 1. Consider an evaluator of type $\theta=\left(u_{g}, \hat{\mu}_{g}, \hat{\tau}_{g}, \hat{\eta}_{g}\right)_{g \in\{M, F\}}$. Observing $v(s, g, \theta), s$ and $g$ for an interval of signals $\mathcal{S}$ such that there exists an $s_{1}, s_{2} \in \mathcal{S}$ with $v\left(s_{1}, g, \theta\right) \neq v\left(s_{2}, g, \theta\right)$ identifies $\bar{s}(\theta, g)$ for each $g \in\{M, F\}$. From Eq. (2), each pair of thresholds $\bar{s}(\theta, M) \neq \bar{s}(\theta, F)$ map into a unique isodiscrimination curve $\left(s_{F}, s_{M}\right)$ with $s_{F} \neq s_{M}$, while $\bar{s}(\theta, M)=\bar{s}(\theta, F)$ map into the isodiscrimination curve corresponding to no discrimination.

Proof of Proposition 2. Consider an evaluator of type $\theta=\left(u_{g}, \hat{\mu}_{g}, \hat{\tau}_{g}, \hat{\eta}_{g}\right)_{g \in\{M, F\}}$ who discriminates against group $F$. This evaluator generates discrimination that lies on isodiscrimination curve $\left(s_{F}, s_{M}\right)=(\bar{s}(\theta, F), \bar{s}(\theta, M))$. Given that $\theta$ discriminates against group $F, s_{F}>s_{M}$. It is immediately apparent from Eq. (2) that there are a continuum of other types that exhibit equivalent discrimination. We next construct types with a single form of partiality.

Part (1): Consider a type $\theta^{\prime}$ with belief neutrality, $\left(\hat{\mu}_{F}^{\prime}, \hat{\tau}_{F}^{\prime}, \hat{\eta}_{F}^{\prime}\right)=\left(\hat{\mu}_{M}^{\prime}, \hat{\tau}_{M}^{\prime}, \hat{\eta}_{M}^{\prime}\right)$. Let $\left(\hat{\mu}^{\prime}, \hat{\tau}^{\prime}, \hat{\eta}^{\prime}\right)$ denote the type's subjective beliefs for a worker from either group. Given preference parameters $\left(u_{F}^{\prime}, u_{M}^{\prime}\right)$, this type hires members of group $g$ with signals above

$$
\left(\frac{\hat{\tau}^{\prime}+\hat{\eta}^{\prime}}{\hat{\eta}^{\prime}}\right) u_{g}^{\prime}-\frac{\hat{\tau}^{\prime}}{\hat{\eta}^{\prime}} \hat{\mu}^{\prime}
$$

This type exhibits equivalent discrimination to $\theta$ if Eq. (4) is equal to $s_{g}$ for each $g \in\{M, F\}$. 
Rearranging terms, this corresponds to preference parameter

$$
u_{g}^{\prime}=\left(\frac{\hat{\eta}^{\prime}}{\hat{\tau}^{\prime}+\hat{\eta}^{\prime}}\right) s_{g}+\frac{\hat{\tau}^{\prime}}{\hat{\tau}^{\prime}+\hat{\eta}^{\prime}} \hat{\mu}^{\prime}
$$

for group $g$. Note $u_{F}^{\prime}>u_{M}^{\prime}$ since $s_{F}>s_{M}$, so there is preference partiality against group $F$.

Part (2): Consider a type $\theta^{\prime}$ with preference neutrality, $u_{F}^{\prime}=u_{M}^{\prime}$ and belief neutrality with respect to concentration and signal precision, $\left(\hat{\tau}_{F}^{\prime}, \hat{\eta}_{F}^{\prime}\right)=\left(\hat{\tau}_{M}^{\prime}, \hat{\eta}_{M}^{\prime}\right)$. Let $\left(u^{\prime}, \hat{\tau}^{\prime}, \hat{\eta}^{\prime}\right)$ denote these common parameters. Given perceived means $\left(\hat{\mu}_{M}^{\prime}, \hat{\mu}_{F}^{\prime}\right)$, this type hires members of group $g$ with signals above

$$
\left(\frac{\hat{\tau}^{\prime}+\hat{\eta}^{\prime}}{\hat{\eta}^{\prime}}\right) u^{\prime}-\frac{\hat{\tau}^{\prime}}{\hat{\eta}^{\prime}} \hat{\mu}_{g}^{\prime} .
$$

This type exhibits equivalent discrimination to $\theta$ if Eq. (5) is equal to $s_{g}$ for each $g \in\{M, F\}$. Rearranging terms, this corresponds to perceived mean

$$
\hat{\mu}_{g}^{\prime}=\left(\frac{\hat{\tau}^{\prime}+\hat{\eta}^{\prime}}{\hat{\tau}^{\prime}}\right) u^{\prime}-\frac{\hat{\eta}^{\prime}}{\hat{\tau}^{\prime}} s_{g}
$$

for group $g$. Note $\hat{\mu}_{F}^{\prime}<\hat{\mu}_{M}^{\prime}$ since $s_{F}>s_{M}$, so there is belief partiality in the form of lower expected productivity against group $F$.

Part (3): Consider a type $\theta^{\prime}$ with preference neutrality, $u_{F}^{\prime}=u_{M}^{\prime}$ and belief neutrality with respect to average productivity and signal precision, $\left(\hat{\mu}_{F}^{\prime}, \hat{\eta}_{F}^{\prime}\right)=\left(\hat{\mu}_{M}^{\prime}, \hat{\eta}_{M}^{\prime}\right)$. Let $\left(u^{\prime}, \hat{\mu}^{\prime}, \hat{\eta}^{\prime}\right)$ denote these common parameters. Given perceived concentration of productivity $\left(\hat{\tau}_{M}^{\prime}, \hat{\tau}_{F}^{\prime}\right)$, this type hires members of group $g$ with signals above

$$
\left(\frac{\hat{\tau}_{g}^{\prime}+\hat{\eta}^{\prime}}{\hat{\eta}^{\prime}}\right) u^{\prime}-\frac{\hat{\tau}_{g}^{\prime}}{\hat{\eta}^{\prime}} \hat{\mu}^{\prime} .
$$

This type exhibits equivalent discrimination to $\theta$ if Eq. (6) is equal to $s_{g}$ for each $g \in\{M, F\}$. Rearranging terms, this corresponds to perceived concentration

$$
\hat{\tau}_{g}^{\prime}=\hat{\eta}^{\prime}\left(\frac{s_{g}-u^{\prime}}{u^{\prime}-\hat{\mu}^{\prime}}\right)
$$

for group $g$. Given $s_{F}>s_{M}, \hat{\eta}^{\prime}\left(s_{F}-u^{\prime}\right)>\hat{\eta}^{\prime}\left(s_{M}-u^{\prime}\right)$. Therefore, whether $\hat{\tau}_{F}^{\prime}$ is greater than or less than $\hat{\tau}_{M}^{\prime}$ depends on the sign of $u^{\prime}-\hat{\mu}^{\prime}$.

If $\theta^{\prime}$ believes the market is lemon-dropping, i.e. $u^{\prime}-\hat{\mu}^{\prime}<0$, then $\hat{\tau}_{F}^{\prime}<\hat{\tau}_{M}^{\prime}$ and a less concentrated perceived productivity distribution generates the discrimination against group $F$. The fatter low productivity tail for $F$ relative to $M$ means that a larger share of workers 
from group $F$ fall below the threshold ex-ante. We also need to check that $\hat{\tau}_{F}^{\prime}>0$ for these to both be a valid precisions. This will be the case for $u^{\prime}>s_{F}$, so that the numerator is also negative. In summary, any type with $\hat{\mu}^{\prime}>s_{F}, u^{\prime} \in\left(s_{F}, \hat{\mu}^{\prime}\right)$ and $\hat{\tau}_{g}^{\prime}=\hat{\eta}^{\prime}\left(\frac{s_{g}-u^{\prime}}{u^{\prime}-\hat{\mu}^{\prime}}\right)$ has belief partiality in the form of lower perceived concentration for group $F$ and exhibits equivalent discrimination to $\theta$.

If $\theta^{\prime}$ believes the market is cherry-picking, i.e. $u^{\prime}-\hat{\mu}^{\prime}>0$, then $\hat{\tau}_{F}^{\prime}>\hat{\tau}_{M}^{\prime}$ and a more concentrated perceived productivity distribution generates the discrimination against group $F$. The thinner high productivity tail for $F$ relative to $M$ means that a smaller share of workers from group $F$ lie above the threshold ex-ante. We also need to check that $\hat{\tau}_{M}^{\prime}>0$ for these to both be valid precisions. This will be the case for $s_{M}>u^{\prime}$, so that the numerator is also positive. In summary, any type with $\hat{\mu}^{\prime}<s_{M}, u^{\prime} \in\left(\hat{\mu}^{\prime}, s_{M}\right)$ and $\hat{\tau}_{g}^{\prime}=\hat{\eta}^{\prime}\left(\frac{s_{g}-u^{\prime}}{u^{\prime}-\hat{\mu}^{\prime}}\right)$ has belief partiality in the form of higher perceived concentration for group $F$ and exhibits equivalent discrimination to $\theta$.

Part (4): Consider a type $\theta^{\prime}$ with preference neutrality, $u_{F}^{\prime}=u_{M}^{\prime}$ and belief neutrality with respect to average productivity and concentration, $\left(\hat{\mu}_{F}^{\prime}, \hat{\tau}_{F}^{\prime}\right)=\left(\hat{\mu}_{M}^{\prime}, \hat{\tau}_{M}^{\prime}\right)$. Let $\left(u^{\prime}, \hat{\mu}^{\prime}, \hat{\tau}^{\prime}\right)$ denote these common parameters. Given perceived signal precision $\left(\hat{\eta}_{M}^{\prime}, \hat{\eta}_{F}^{\prime}\right)$, this type hires members of group $g$ with signals above

$$
\left(\frac{\hat{\tau}^{\prime}+\hat{\eta}_{g}^{\prime}}{\hat{\eta}_{g}^{\prime}}\right) u^{\prime}-\frac{\hat{\tau}^{\prime}}{\hat{\eta}_{g}^{\prime}} \hat{\mu}^{\prime} .
$$

This type exhibits equivalent discrimination to $\theta$ if Eq. (7) is equal to $s_{g}$ for each $g \in\{M, F\}$. Rearranging terms, this corresponds to perceived signal precision

$$
\hat{\eta}_{g}^{\prime}=\hat{\tau}^{\prime}\left(\frac{u^{\prime}-\hat{\mu}^{\prime}}{s_{g}-u^{\prime}}\right)
$$

for group $g$. Given $s_{F}>s_{M}, s_{F}-u^{\prime}>s_{M}-u^{\prime}$. We need $\hat{\eta}_{g}^{\prime}>0$ for each $g$ in order for these to be valid precisions. This is the case when (i) $u^{\prime}-\hat{\mu}^{\prime}<0$ and $s_{F}-u^{\prime}<0$, which also implies $s_{M}-u^{\prime}<0$, or (ii) $u^{\prime}-\hat{\mu}^{\prime}>0$ and $s_{M}-u^{\prime}>0$, which also implies $s_{F}-u^{\prime}>0$.

First consider case (i). In this case, $\theta^{\prime}$ believes the market is lemon-dropping since $u^{\prime}<\hat{\mu}^{\prime}$. Further, $0>s_{F}-u^{\prime}>s_{M}-u^{\prime} \Rightarrow 1 /\left(s_{M}-u^{\prime}\right)>1 /\left(s_{F}-u^{\prime}\right) \Rightarrow\left(u^{\prime}-\hat{\mu}^{\prime}\right) /\left(s_{M}-u^{\prime}\right)<$ $\left(u^{\prime}-\hat{\mu}^{\prime}\right) /\left(s_{F}-u^{\prime}\right)$. Therefore, $\hat{\eta}_{M}^{\prime}<\hat{\eta}_{F}^{\prime}$ and a higher perceived signal precision generates the discrimination against group $F$. In summary, any type with $\hat{\mu}^{\prime}>s_{F}, u^{\prime} \in\left(s_{F}, \hat{\mu}^{\prime}\right)$ and $\hat{\eta}_{g}^{\prime}=\hat{\tau}^{\prime}\left(\frac{u^{\prime}-\hat{\mu}^{\prime}}{s_{g}-u^{\prime}}\right)$ has belief partiality in the form of higher perceived signal precision for group $F$ and exhibits equivalent discrimination to $\theta$. 
Next consider case (ii). In this case, $\theta^{\prime}$ believes the market is cherry-picking since $u^{\prime}>\hat{\mu}^{\prime}$. Further, $s_{F}-u^{\prime}>s_{M}-u^{\prime}>0 \Rightarrow 1 /\left(s_{M}-u^{\prime}\right)>1 /\left(s_{F}-u^{\prime}\right) \Rightarrow\left(u^{\prime}-\hat{\mu}^{\prime}\right) /\left(s_{M}-u^{\prime}\right)>$ $\left(u^{\prime}-\hat{\mu}^{\prime}\right) /\left(s_{F}-u^{\prime}\right)$. Therefore, $\hat{\eta}_{M}^{\prime}>\hat{\eta}_{F}^{\prime}$ and a lower perceived signal precision generates the discrimination against group $F$. In summary, any type with $\hat{\mu}^{\prime}<s_{F}, u^{\prime} \in\left(\hat{\mu}^{\prime}, s_{M}\right)$ and $\hat{\eta}_{g}^{\prime}=\hat{\tau}^{\prime}\left(\frac{u^{\prime}-\hat{\mu}^{\prime}}{s_{g}-u^{\prime}}\right)$ has belief partiality in the form of lower perceived signal precision for group $F$ and exhibits equivalent discrimination to $\theta$.

Proof of Observation 2. Suppose the evaluator has type $\theta=\left(u_{g}, \hat{\mu}_{g}, \hat{\tau}_{g}, \hat{\eta}_{g}\right)_{g \in\{M, F\}}$. This evaluator exhibits discrimination that lies on isodiscrimination curve $\left(s_{F}, s_{M}\right)=(\bar{s}(\theta, F), \bar{s}(\theta, M))$. Suppose the researcher identifies the isodiscrimination curve $\left(s_{F}, s_{M}\right)$ and the true productivity and signal distributions $\left(\mu_{g}, \tau_{g}, \eta_{g}\right)$ for each group $g$. Under the assumption of accurate beliefs, i.e. $\left(\hat{\mu}_{g}, \hat{\tau}_{g}, \hat{\eta}_{g}\right)=\left(\mu_{g}, \tau_{g}, \eta_{g}\right)$, solving Eq. (2) for $u_{g}$ uniquely identifies the preference parameters $\left(u_{F}, u_{M}\right)$ as

$$
u_{g}=\left(\frac{\eta_{g}}{\tau_{g}+\eta_{g}}\right) s_{g}+\left(\frac{\tau_{g}}{\tau_{g}+\eta_{g}}\right) \mu_{g} .
$$

Therefore, the evaluator's type is identified.

Proof of Observation 3. Suppose the evaluator has type $\theta=\left(u_{g}, \hat{\mu}_{g}, \hat{\tau}_{g}, \hat{\eta}_{g}\right)_{g \in\{M, F\}}$. This evaluator exhibits discrimination that lies on isodiscrimination curve $\left(s_{F}, s_{M}\right)=(\bar{s}(\theta, F), \bar{s}(\theta, M))$. Suppose the researcher identifies the isodiscrimination curve $\left(s_{F}, s_{M}\right)$ and the true productivity and signal distributions $\left(\mu_{g}, \tau_{g}, \eta_{g}\right)$ for each group $g$. From Eq. (2), it is clear that when beliefs may be inaccurate, this provides no additional information about $\left(u_{g}, \hat{\mu}_{g}, \hat{\tau}_{g}, \hat{\eta}_{g}\right)$ for each group $g$ - any type that satisfies Eq. (2) for the observed isodiscrimination curve can exhibit the observed behavior.

Proof of Observation 4. Given true productivity and signal distributions $\left(\mu_{g}, \tau_{g}, \eta_{g}\right)_{g \in\{M, F\}}$, suppose the evaluator has type $\theta=\left(u_{g}, \hat{\mu}_{g}, \hat{\tau}_{g}, \hat{\eta}_{g}\right)_{g \in\{M, F\}}$ with inaccurate beliefs, $\left(\hat{\mu}_{g}, \hat{\tau}_{g}, \hat{\eta}_{g}\right) \neq$ $\left(\mu_{g}, \tau_{g}, \eta_{g}\right)$. This evaluator exhibits discrimination that lies on isodiscrimination curve $\left(s_{F}, s_{M}\right)=$ $(\bar{s}(\theta, F), \bar{s}(\theta, M))$. Suppose a researcher identifies the isodiscrimination curve $\left(s_{F}, s_{M}\right)$ and the true productivity and signal distributions $\left(\mu_{g}, \tau_{g}, \eta_{g}\right)$ for each group $g$. When the researcher assumes belief are accurate, i.e. the evaluator is a type $\theta^{\prime}$ with beliefs $\left(\hat{\mu}_{g}^{\prime}, \hat{\tau}_{g}^{\prime}, \hat{\eta}_{g}^{\prime}\right)=$ $\left(\mu_{g}, \tau_{g}, \eta_{g}\right)$, then from Observation 2 , the researcher concludes that the evaluator has preference parameter

$$
u_{g}^{\prime}=\left(\frac{\eta_{g}}{\tau_{g}+\eta_{g}}\right) s_{g}+\left(\frac{\tau_{g}}{\tau_{g}+\eta_{g}}\right) \mu_{g}
$$


In contrast, the the true preference parameter satisfies

$$
u_{g}=\left(\frac{\hat{\eta}_{g}}{\hat{\tau}_{g}+\hat{\eta}_{g}}\right) s_{g}+\left(\frac{\hat{\tau}_{g}}{\hat{\tau}_{g}+\hat{\eta}_{g}}\right) \hat{\mu}_{g}
$$

When beliefs are inaccurate, this identified preference parameter is equal to the true parameter, $u_{g}^{\prime}=u_{g}$, if and only if

$$
\mu_{g}=\left(\frac{\tau_{g}+\eta_{g}}{\tau_{g}}\right)\left[\left(\frac{\hat{\eta}_{g}}{\hat{\tau}_{g}+\hat{\eta}_{g}}\right) s_{g}-\left(\frac{\eta_{g}}{\tau_{g}+\eta_{g}}\right) s_{g}+\left(\frac{\hat{\tau}_{g}}{\hat{\tau}_{g}+\hat{\eta}_{g}}\right) \hat{\mu}_{g}\right]
$$

Therefore, the preference parameter is misidentified for a generic set of true beliefs $\left(\mu_{g}, \tau_{g}, \eta_{g}\right)$ and evaluator types $\theta=\left(u_{g}, \hat{\mu}_{g}, \hat{\tau}_{g}, \hat{\eta}_{g}\right)_{g \in\{M, F\}}, u_{g}^{\prime} \neq u_{g}$.

Let $\theta^{*}=\left(u_{g}, \mu_{g}, \tau_{g}, \eta_{g}\right)_{g \in\{M, F\}}$ denote the type with accurate beliefs and the same preferences as $\theta$. Suppose type $\theta$ 's inaccurate beliefs increase discrimination against group $F$, i.e. $\bar{s}(\theta, F) \geq \bar{s}\left(\theta^{*}, F\right)$ and $\bar{s}\left(\theta^{*}, M\right) \geq \bar{s}(\theta, M)$ with at least one strict inequality. Then given the observed isodiscrimination curve is consistent with the true type, i.e. $s_{F}=\bar{s}(\theta, F)$, and from the expression for $\bar{s}\left(\theta^{*}, F\right)$ in Eq. (1),

$$
s_{F} \geq \frac{\tau_{F}+\eta_{F}}{\eta_{F}} u_{F}-\frac{\tau_{F}}{\eta_{F}} \mu_{F}
$$

Combining Eqs. (9) and (12)

$$
u_{F}^{\prime}=\left(\frac{\eta_{F}}{\tau_{F}+\eta_{F}}\right) s_{F}+\left(\frac{\tau_{F}}{\tau_{F}+\eta_{F}}\right) \mu_{F} \geq u_{F} .
$$

Similarly, $u_{M}^{\prime} \leq u_{M}$, with a strict inequality for at least one of the expressions. Therefore, the researcher overestimates the preference parameter for group $F$ and/or underestimates the preference parameter for group $M$, leading her to overestimate the preference partiality against group $F$. The proof for the case of $\theta$ 's inaccurate beliefs decreasing discrimination is analogous.

Proof of Observation 5. Suppose the researcher identifies the isodiscrimination curve $\left(s_{F}, s_{M}\right)$ and the true productivity and signal distributions $\left(\mu_{g}, \tau_{g}, \eta_{g}\right)$ for each group $g$. From Eq. (2), for any $u \in \mathbb{R}$, the corresponding accurate statistical discriminator with preferences 
$u_{M}=u_{F}=u$ lies on isodiscrimination curve $\left(s_{F}^{\prime}, s_{M}^{\prime}\right)$ with

$$
s_{g}^{\prime}=\left(\frac{\tau_{g}+\eta_{g}}{\eta_{g}}\right) u-\frac{\tau_{g}}{\eta_{g}} \mu_{g}
$$

If $\frac{\tau_{M} \mu_{M}+\eta_{M} s_{M}}{\tau_{M}+\eta_{M}} \neq \frac{\tau_{F} \mu_{F}+\eta_{F} s_{F}}{\tau_{F}+\eta_{F}}$, then there is no $u$ such that $\left(s_{F}^{\prime}, s_{M}^{\prime}\right)=\left(s_{F}, s_{M}\right)$, i.e. an accurate statistical discriminator exhibits discrimination that is consistent with the observed isodiscrimination curve.

Proof of Observation 6. Suppose the evaluator has type $\theta=\left(u_{g}, \hat{\mu}_{g}, \hat{\tau}_{g}, \hat{\eta}_{g}\right)_{g \in\{M, F\}}$. This evaluator exhibits discrimination that lies on isodiscrimination curve $\left(s_{F}, s_{M}\right)=(\bar{s}(\theta, F), \bar{s}(\theta, M))$. Suppose the researcher identifies the isodiscrimination curve $\left(s_{F}, s_{M}\right)$ and the perceived productivity and signal distributions $\left(\hat{\mu}_{g}, \hat{\tau}_{g}, \hat{\eta}_{g}\right)$ for each group $g$. Solving Eq. (2) for $u_{g}$ uniquely identifies the preference parameters $\left(u_{F}, u_{M}\right)$ as

$$
u_{g}=\left(\frac{\hat{\eta}_{g}}{\hat{\tau}_{g}+\hat{\eta}_{g}}\right) s_{g}+\left(\frac{\hat{\tau}_{g}}{\hat{\tau}_{g}+\hat{\eta}_{g}}\right) \hat{\mu}_{g}
$$

Therefore, the evaluator's type is identified.

Proof of Proposition 3. Given a signal with precision $\eta>0$, observing $x \geq 1$ draws of the signal is equivalent to observing a single signal that is normally distributed with precision

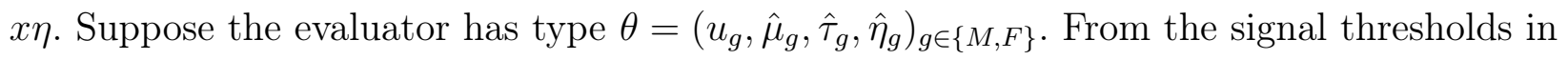
Eq. (1), if a type $\theta^{\prime}=\left(u_{g}^{\prime}, \hat{\mu}_{g}^{\prime}, \hat{\tau}_{g}^{\prime}, \hat{\eta}_{g}^{\prime}\right)_{g \in\{M, F\}}$ exhibits equivalent discrimination to $\theta$ when observing $x \geq 1$ signal draws, then

$$
\frac{\hat{\tau}_{g} u_{g}+x \hat{\eta}_{g} u_{g}-\hat{\tau}_{g} \hat{\mu}_{g}}{x \hat{\eta}_{g}}=\frac{\hat{\tau}_{g}^{\prime} u_{g}^{\prime}+x \hat{\eta}_{g}^{\prime} u_{g}^{\prime}-\hat{\tau}_{g}^{\prime} \hat{\mu}_{g}^{\prime}}{x \hat{\eta}_{g}^{\prime}}
$$

for $g \in\{M, F\}$. Rearranging terms, this is equivalent to

$$
\frac{\hat{\tau}_{g}\left(u_{g}-\hat{\mu}_{g}\right)}{\hat{\eta}_{g}}-\frac{\hat{\tau}_{g}^{\prime}\left(u_{g}^{\prime}-\hat{\mu}_{g}^{\prime}\right)}{\hat{\eta}_{g}^{\prime}}=x\left(u_{g}^{\prime}-u_{g}\right) .
$$

Suppose $\theta^{\prime}$ exhibits equivalent discrimination to $\theta$ when observing $x_{1} \geq 1$ and $x_{2}>x_{1}$ signal draws. Then Eq. (17) must be simultaneously satisfied at $x=x_{1}$ and $x=x_{2}$. Since the left hand side of Eq. (17) is independent of $x$, this requires $x_{1}\left(u_{g}^{\prime}-u_{g}\right)=x_{2}\left(u_{g}^{\prime}-u_{g}\right)$. Given $x_{1} \neq x_{2}$, it must be that $u_{g}^{\prime}=u_{g}$. Therefore, all types that exhibit equivalent discrimination to $\theta$ in both informational treatments have the same preferences as $\theta, u_{F}^{\prime}=u_{F}$ and $u_{M}^{\prime}=u_{M}$. 
The right hand side of Eq. (17) is equal to zero for these types. Therefore, all types that exhibit equivalent discrimination to $\theta$ in both informational treatments have beliefs and preferences that satisfy

$$
\frac{\hat{\tau}_{g}\left(u_{g}-\hat{\mu}_{g}\right)}{\hat{\eta}_{g}}=\frac{\hat{\tau}_{g}^{\prime}\left(u_{g}-\hat{\mu}_{g}^{\prime}\right)}{\hat{\eta}_{g}^{\prime}}
$$

Solving for $\hat{\mu}_{g}^{\prime}$, the set of types that exhibit equivalent discrimination to $\theta$ corresponds to

$$
\left\{\theta^{\prime}=\left(u_{g}, \hat{\mu}_{g}^{\prime}, \hat{\tau}_{g}^{\prime}, \hat{\eta}_{g}^{\prime}\right)_{g \in\{M, F\}} \mid \hat{\mu}_{g}^{\prime}=u_{g}-\frac{\hat{\tau}_{g} / \hat{\eta}_{g}}{\hat{\tau}_{g}^{\prime} / \hat{\eta}_{g}^{\prime}}\left(u_{g}-\hat{\mu}_{g}\right)\right\}
$$

If $\hat{\mu}_{g}^{\prime}=\hat{\mu}_{g}$, this corresponds to the set of types that preserve the ratio of the precisions. If the perceived precisions are equal across groups, i.e. $\hat{\tau}_{g}^{\prime}=\hat{\tau}_{g}$ and $\hat{\eta}_{g}^{\prime}=\hat{\eta}_{g}$, then it must be that $\hat{\mu}_{g}^{\prime}=\hat{\mu}_{g}$, which means $\theta^{\prime}=\theta$. In this case, $\theta$ is identified from observing discrimination for two informational treatments.

Suppose $\theta^{\prime}$ exhibits equivalent discrimination to $\theta$ for two informational treatments, denoted $x_{1}$ and $x_{2}$. Then $u_{F}^{\prime}=u_{F}$ and $u_{M}^{\prime}=u_{M}$, so Eq. (17) is satisfied for any other informational treatment $x \notin\left\{x_{1}, x_{2}\right\}$, i.e. $\theta^{\prime}$ also exhibits equivalent discrimination for informational treatment $x$. Therefore, if type $\theta^{\prime}$ exhibits equivalent discrimination to $\theta$ for two informational treatments, $\theta^{\prime}$ also exhibits equivalent discrimination to $\theta$ for all possible informational treatments.

Proof of Observation 7. Suppose the evaluator has type $\theta=\left(u_{g}, \hat{\mu}_{g}, \hat{\tau}_{g}, \hat{\eta}_{g}\right)_{g \in\{M, F\}}$. Let $\theta(x)=\left(u_{g}, \hat{\mu}_{g}, \hat{\tau}_{g}, x \hat{\eta}_{g}\right)_{g \in\{M, F\}}$ denote a type with the same preferences and beliefs about the productivity distribution as $\theta$ and perceived signal precision $x \hat{\eta}_{g}$. When type $\theta$ observes $x$ draws of the signal, it behaves as if it is type $\theta(x)$. Therefore, it exhibits discrimination that lies on isodiscrimination curve $(\bar{s}(\theta(x), F), \bar{s}(\theta(x), M))$. Suppose the researcher identifies the isodiscrimination curves for $\theta$ when it observes $x_{1}$ and $x_{2} \neq x_{1}$ signal draws, $\left(s_{F, 1}, s_{M, 1}\right) \equiv$ $\left(\bar{s}\left(\theta\left(x_{1}\right), F\right), \bar{s}\left(\theta\left(x_{1}\right), M\right)\right)$ and $\left(s_{F, 2}, s_{M, 2}\right) \equiv\left(\bar{s}\left(\theta\left(x_{2}\right), F\right), \bar{s}\left(\theta\left(x_{2}\right), M\right)\right)$. Then from Eq. (2), we know

$$
\frac{\hat{\tau}_{g}+x_{i} \hat{\eta}_{g}}{x_{i} \hat{\eta}_{g}} u_{g}-\frac{\hat{\tau}_{g}}{x_{i} \hat{\eta}_{g}} \hat{\mu}_{g}=s_{g, i}
$$


for $i=1,2$. Rearranging terms,

$$
\left(\frac{\hat{\tau}_{g}}{\hat{\eta}_{g}}+x_{i}\right) u_{g}=x_{i} s_{g, i}+\frac{\hat{\tau}_{g}}{\hat{\eta}_{g}} \hat{\mu}_{g} .
$$

Subtracting Eq. (19) evaluated at $x_{2}$ from Eq. (19) evaluated at $x_{1}$ yields

$$
\begin{aligned}
\left(x_{1}-x_{2}\right) u_{g} & =x_{1} s_{g, 1}-x_{2} s_{g, 2} \\
\Rightarrow u_{g} & =\frac{x_{1} s_{g, 1}-x_{2} s_{g, 2}}{x_{1}-x_{2}} .
\end{aligned}
$$

This uniquely identifies the evaluator's preferences. However, as shown in Proposition 3, multiple sets of beliefs $\left(\hat{\mu}_{g}, \hat{\tau}_{g}, \hat{\tau}_{g}\right)$ can satisfy Eq. (19) for $x_{1}$ and $x_{2}$. Therefore, the evaluator's beliefs are not identified.

\section{Appendix B. Experimental Design}

Our experimental design includes two separate, pre-registered surveys: (1) a work task (math quiz) performed by 589 Amazon Mechanical Turk subjects (MTurkers), who comprise the prospective "workers" for the second survey, (2) a hiring task in which each of 577 different MTurkers, who comprise the "employers," stated a wage (willingness to pay) for 20 prospective worker profiles. ${ }^{16}$ The second survey also contains a belief elicitation and an information intervention followed by a second hiring task. The full surveys are in the Online Appendix. We describe the experimental design and provide summary statistics below.

Survey 1 (Work Task): We recruited 589 subjects from MTurk on February 23, 2018 for the first survey. ${ }^{17}$ The survey was posted with the title "Math Questions and Demographics" and the description "A 20-minute task of answering math questions." We paid $\$ 2$ (i.e. a projected $\$ 6 /$ hour wage) and recruited a subject pool of 392 from the United States and 197 from India, all of whom had completed at least 500 prior tasks and had an $80 \%$ or higher approval rate for these tasks. ${ }^{18}$ After starting the survey, subjects were informed that

\footnotetext{
${ }^{16}$ We pre-registered the study on AsPredicted.org. There are two minor differences between the preregistration plan and the actual study. First, we pre-registered that we would recruit 400 employers in the hiring task survey, but decided to recruit closer to 600 . Second, we did not pre-register sample restrictions due to completing the task too quickly or slowly. We dropped 12 subjects in the work task survey and 5 in the hiring task survey due to these restrictions.

${ }^{17}$ We received 604 responses in total, but dropped 12 responses that corresponded to the top $1 \%(<227$ seconds) and bottom 1\% (> 3274 seconds) in terms of survey duration. Of the remaining 592 responses, we dropped 3 whose Qualtrics survey responses could not be matched to their MTurk records, leaving 589 final respondents.

${ }^{18}$ This geographic restriction is based on the addresses MTurkers used to register on Amazon. The survey
} 
they would first answer demographic questions and then answer 50 multiple choice math questions. They were told that their performance would not affect their payment, and were asked not to use a calculator or any outside help, but just to do their best. This was followed by seven questions that provided the information used for their profiles in the second survey: favorite color, favorite movie, coffee vs. tea preference, age, gender, favorite subject in high school, and favorite sport. The math test included a mix of arithmetic (e.g. " $5 * 6 * 7=$ ?"), algebra (e.g. "If $(y+9) *\left(y^{2}-121\right)=0$, then which of the following cannot be y?"), and more conceptual questions (e.g. "Which of the following is not a prime number?"). Finally, subjects were thanked for their participation and informed that they may receive a small bonus based on a different experiment, for reasons unrelated to their performance on the task. We describe the basis for such bonuses in the description of Survey 2.

The purpose of the first survey was to create a bank of "workers" who could be hired by the "employers" in the second survey. This novel design has several advantages over the existing paradigms for studying discrimination in the field. First, in contrast to correspondence studies, we did not employ deception at any point - all profiles shown to employers corresponded to actual workers who would in fact be paid as described in the following paragraph. However, similar to a correspondence study, we were able to control the information seen by an employer about a prospective worker by constructing worker profiles that included information that is ostensibly relevant for animus and/or beliefs about productivity (e.g. age, gender, and nationality), as well as other irrelevant information (e.g. tea preference). The irrelevant information serves as a placebo test and ensures that the relevant information is not the only salient information provided to the employer (this mimics the irrelevant information contained on a CV). Finally, instead of the coarse measures of discrimination used in many other studies (e.g. callback or stop rates), we elicit relatively continuous and precise measures of productivity and discrimination that are tightly linked.

Survey 2: We recruited 577 different MTurk subjects on February 26, 2018. We used the same hiring criteria as the first survey (392 from U.S., 185 from India, $>=80 \%$ approval rate). ${ }^{19}$ The survey was posted with the title "20-Minute Survey about Decision-Making" and the description "20-Minute Survey about Decision-Making." We paid $\$ 2$ (i.e. a projected $\$ 6 /$ hour wage). Subjects were first asked to report their gender, age, and education level. Subjects were then presented with the first hiring task portion of the survey.

was posted as two tasks on MTurk, with one only eligible for Indian workers and one only eligible for U.S. workers.

${ }^{19}$ We recruited 587 subjects in total, but dropped 7 whose surveys were completed in under 300 seconds and 3 whose stimuli (the profiles they evaluated) could not be matched to the first survey. 
First Hiring Task: We informed subjects that we had previously paid other subjects ("workers") to answer 50 math questions, showed them five examples of the math questions, and told them that on average, participants answered 36.95 out of 50 questions correctly. They were then told that they would act as an employer and hire one of these workers by stating a wage (paid as a bonus to the worker). In return, they would receive a payment based on how many questions their hired worker answered correctly. This was followed by a more detailed description of the assignment. Each "employer" would view 20 profiles of potential workers and state the highest wage (between 0 to 50 cents) they were willing to pay to each worker. The employer would be paid 1 cent for each question answered correctly by the hired worker. We next described the mechanism (Becker-Degroot-Marschak) used to assign payment. We would randomly select a profile from the 20 potential workers. We would then draw a random number from 0 to 50. If the wage the employer stated for the worker was equal or greater than that number, then the worker would receive the random number as a bonus and the employer would receive a "profit" equal to the worker's performance minus the random number. If instead the employer stated a wage for the worker that was lower than the random number, then neither the worker nor the employer would receive a payment.

To ensure comprehension, we showed subjects an example profile (see Fig. B1) and stated wage. We gave examples of actual performance and randomly generated numbers that would produce positive profit, negative profit, and no hiring. Having highlighted the possibility of negative profit, we then noted that all employers would automatically be paid a $\$ 0.50$ bonus in addition to any money made through the hiring task, so that no employers would owe money. Finally, we ran a comprehension check with the same example profile, a specific wage (43), a random number (18), and an actual performance (10). We required the employer to correctly state how many cents they would have to pay the worker (18) and how many cents the employer would be paid before subtracting off the amount they would pay the worker (10). ${ }^{20}$ Finally, employers were presented with a second wage (15), and answered the same questions. They were then presented with 20 profiles, each randomly selected with replacement from the bank of 589 profiles produced by the first survey.

\footnotetext{
${ }^{20}$ Entering an incorrect an answer would generate a pop-up with "Wrong Answer" and restrict the individual from moving to the next page.
} 
Figure B1. Example Profile Used in First Hiring Task Description

\begin{tabular}{|l|l|} 
Country: & United States \\
Gender: & Female \\
Age: & 63 \\
Favorite High School Subject: & English \\
Favorite Sport: & Gymnastics \\
Favorite Color: & Sea Green \\
Favorite Movie: & Overboard \\
Prefers Coffee/Tea: & Tea \\
\hline
\end{tabular}

Belief Elicitation Task: Next, subjects were randomly assigned to one of two different conditions: an incentivized or un-incentivized belief elicitation. Across both conditions, subjects were reminded that the full sample answered 36.95 out of 50 questions correctly. They were then asked to answer six questions of the form, "On average, how many math questions out of 50 do you think X answered correctly?" where X corresponded to the groups "women", "men", "people from the United States", "people from India", "people below or at the age of 33," and "people above the age of 33."

In the incentivized condition, prior to the six questions, subjects were told that they could earn a significant bonus for an accurate prediction. One of the six questions would be randomly selected and they would be paid $\$ 5$ minus their deviation from the question (bounded below by $\$ 0$ ). For example, if they answered 40 and the true average was 37, they would receive a $\$ 2$ bonus. Finally, they were asked to "please answer the questions as carefully as possible so that you can potentially win a large bonus."

Information Intervention \& Second Hiring Task: After completing the belief elicitation, subjects were shown the correct answer for all six groups: women (35.28), men (38.32), people from the U.S. (37.14), people from India (36.58), people below or at the age of 33 (37.10), and people above the age of 33 (36.79). Following this information, we stated, "Now that you have learned those facts, we would like you to work on 10 more profiles." We noted that, as in the first hiring task, we would randomly select one profile and a number, and pay bonus and wages accordingly (with an additional $\$ 0.50$ automatic bonus to ensure no negative payments). After employers reviewed the 10 additional worker profiles, we thanked them for their participation, noted that we would calculate bonuses and pay them within a 
week, and allowed subjects the option to leave comments.

Summary Statistics: Table B1 provides summary statistics for the full sample of subjects that completed surveys 1 and 2 (Column (1)), as well as these statistics for each of the 6 demographic groups used in the second survey. On average, the work task (survey 1) took subjects 19 minutes to complete, while the hiring task took 23 minutes. There is variation in this timing across groups. Subjects from the U.S. took an average of 19 minutes to complete the hiring task, while subjects from India took 31.60 minutes; a difference also reflected in their median times (15.8 vs. 25.6). Another large difference between the U.S. and India samples is the average age of participants; the average Indian subject in the work task is approximately 8 years younger than the average American subject. This gap shrinks to 4 years for the hiring task. The Indian sample also skews more male than the U.S. sample ( $68.5 \%$ vs. $48.2 \%$ and $76.8 \%$ vs. $51.4 \%$ for survey 1 and 2 , respectively) and is more likely to have a college education or above ( $90.3 \%$ vs. $56 \%$ in survey 2 ; the question was not asked in survey 1). While we primarily focus on simple comparisons between each demographic group, these observed differences motivate our use of multivariate regressions as well. 
Table B1. Summary Statistics

\begin{tabular}{|c|c|c|c|c|c|c|c|}
\hline & $\begin{array}{c}\text { Total } \\
(1)\end{array}$ & $\begin{array}{c}\text { Male } \\
(2)\end{array}$ & $\begin{array}{c}\text { Female } \\
(3)\end{array}$ & $\begin{array}{l}\text { US } \\
(4)\end{array}$ & $\begin{array}{c}\text { India } \\
(5)\end{array}$ & $\begin{array}{c}\text { Under } 33 \\
(6)\end{array}$ & $\begin{array}{c}\text { Over } 33 \\
(7)\end{array}$ \\
\hline \multicolumn{8}{|l|}{ Panel A: Worker } \\
\hline Trivia Score & $\begin{array}{l}36.95 \\
(8.73)\end{array}$ & $\begin{array}{l}38.32 \\
(8.52)\end{array}$ & $\begin{array}{l}35.28 \\
(8.70)\end{array}$ & $\begin{array}{l}37.14 \\
(8.93)\end{array}$ & $\begin{array}{l}36.58 \\
(8.31)\end{array}$ & $\begin{array}{l}37.10 \\
(8.55)\end{array}$ & $\begin{array}{l}36.79 \\
(8.94)\end{array}$ \\
\hline Survey Duration (Minutes) & $\begin{array}{c}18.82 \\
(10.39)\end{array}$ & $\begin{array}{c}19.03 \\
(10.52)\end{array}$ & $\begin{array}{c}18.56 \\
(10.25)\end{array}$ & $\begin{array}{l}16.19 \\
(8.12)\end{array}$ & $\begin{array}{c}24.04 \\
(12.31)\end{array}$ & $\begin{array}{c}20.25 \\
(11.82)\end{array}$ & $\begin{array}{l}17.18 \\
(8.20)\end{array}$ \\
\hline Prefer Tea $($ Yes $=1)$ & $\begin{array}{c}0.39 \\
(0.49)\end{array}$ & $\begin{array}{c}0.38 \\
(0.49)\end{array}$ & $\begin{array}{c}0.41 \\
(0.49)\end{array}$ & $\begin{array}{c}0.37 \\
(0.48)\end{array}$ & $\begin{array}{c}0.44 \\
(0.50)\end{array}$ & $\begin{array}{c}0.42 \\
(0.49)\end{array}$ & $\begin{array}{c}0.36 \\
(0.48)\end{array}$ \\
\hline Age (Worker) & $\begin{array}{c}35.89 \\
(11.57)\end{array}$ & $\begin{array}{c}35.30 \\
(11.27)\end{array}$ & $\begin{array}{c}36.62 \\
(11.91)\end{array}$ & $\begin{array}{c}38.55 \\
(12.16)\end{array}$ & $\begin{array}{l}30.61 \\
(8.01)\end{array}$ & $\begin{array}{l}27.38 \\
(3.50)\end{array}$ & $\begin{array}{l}45.61 \\
(9.76)\end{array}$ \\
\hline Female $($ Yes $=1)$ & $\begin{array}{c}0.45 \\
(0.50)\end{array}$ & $\begin{array}{c}0.00 \\
(0.00)\end{array}$ & $\begin{array}{c}1.00 \\
(0.00)\end{array}$ & $\begin{array}{c}0.52 \\
(0.50)\end{array}$ & $\begin{array}{c}0.32 \\
(0.47)\end{array}$ & $\begin{array}{c}0.43 \\
(0.50)\end{array}$ & $\begin{array}{c}0.48 \\
(0.50)\end{array}$ \\
\hline From India (Yes=1) & $\begin{array}{c}0.33 \\
(0.47)\end{array}$ & $\begin{array}{c}0.42 \\
(0.49)\end{array}$ & $\begin{array}{c}0.23 \\
(0.42)\end{array}$ & $\begin{array}{c}0.00 \\
(0.00)\end{array}$ & $\begin{array}{c}1.00 \\
(0.00)\end{array}$ & $\begin{array}{c}0.47 \\
(0.50)\end{array}$ & $\begin{array}{c}0.18 \\
(0.39)\end{array}$ \\
\hline \# Observations & 589 & 324 & 265 & 392 & 197 & 314 & 275 \\
\hline Panel B: Employer & & & & & & & \\
\hline Survey Duration (Minutes) & $\begin{array}{c}23.09 \\
(17.23)\end{array}$ & $\begin{array}{c}23.59 \\
(15.57)\end{array}$ & $\begin{array}{c}22.37 \\
(19.43)\end{array}$ & $\begin{array}{c}19.08 \\
(11.70)\end{array}$ & $\begin{array}{c}31.60 \\
(23.04)\end{array}$ & $\begin{array}{c}22.53 \\
(19.00)\end{array}$ & $\begin{array}{c}23.87 \\
(14.44)\end{array}$ \\
\hline College Education or Above & $\begin{array}{c}0.67 \\
(0.47)\end{array}$ & $\begin{array}{c}0.70 \\
(0.46)\end{array}$ & $\begin{array}{c}0.62 \\
(0.49)\end{array}$ & $\begin{array}{c}0.56 \\
(0.50)\end{array}$ & $\begin{array}{c}0.90 \\
(0.30)\end{array}$ & $\begin{array}{c}0.67 \\
(0.47)\end{array}$ & $\begin{array}{c}0.67 \\
(0.47)\end{array}$ \\
\hline Age (Employer) & $\begin{array}{c}34.36 \\
(11.02)\end{array}$ & $\begin{array}{l}32.66 \\
(9.92)\end{array}$ & $\begin{array}{c}36.88 \\
(12.07)\end{array}$ & $\begin{array}{c}35.73 \\
(11.63)\end{array}$ & $\begin{array}{l}31.46 \\
(8.96)\end{array}$ & $\begin{array}{l}27.09 \\
(3.59)\end{array}$ & $\begin{array}{l}44.36 \\
(9.91)\end{array}$ \\
\hline Female $(\mathrm{Yes}=1)$ & $\begin{array}{c}0.40 \\
(0.49)\end{array}$ & $\begin{array}{c}0.00 \\
(0.00)\end{array}$ & $\begin{array}{c}1.00 \\
(0.00)\end{array}$ & $\begin{array}{c}0.49 \\
(0.50)\end{array}$ & $\begin{array}{c}0.23 \\
(0.42)\end{array}$ & $\begin{array}{c}0.34 \\
(0.47)\end{array}$ & $\begin{array}{c}0.49 \\
(0.50)\end{array}$ \\
\hline From India $(Y e s=1)$ & $\begin{array}{c}0.32 \\
(0.47)\end{array}$ & $\begin{array}{c}0.41 \\
(0.49)\end{array}$ & $\begin{array}{c}0.19 \\
(0.39)\end{array}$ & $\begin{array}{c}0.00 \\
(0.00)\end{array}$ & $\begin{array}{c}1.00 \\
(0.00)\end{array}$ & $\begin{array}{c}0.40 \\
(0.49)\end{array}$ & $\begin{array}{c}0.29 \\
(0.41)\end{array}$ \\
\hline \# Observations & 577 & 344 & 233 & 392 & 185 & 334 & 243 \\
\hline
\end{tabular}

Notes: Standard deviations in parentheses. One observation per worker (survey 1) or employer (survey 2). 


\section{Appendix C. Additional Tables and Figures}

Figure C2. Kernel Densities of Productivities (Trivia Scores) by Group

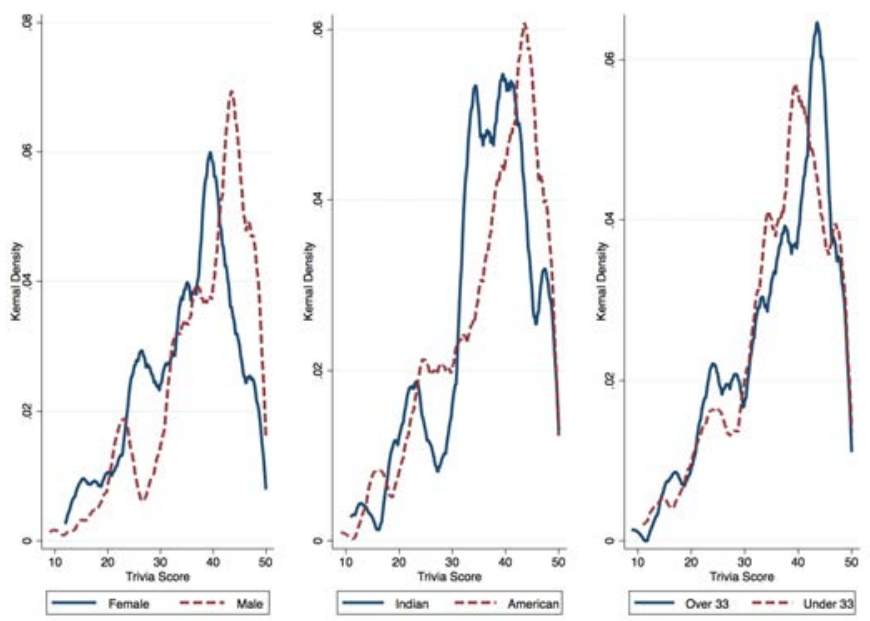

Table C2. Effects of Large Incentives for Accurate Predictions

\begin{tabular}{|c|c|c|c|c|}
\hline & \multicolumn{2}{|c|}{ Incentivized? } & \multirow[t]{2}{*}{ Diff. } & \multirow[t]{2}{*}{ p-val } \\
\hline & No & Yes & & \\
\hline & (1) & $(2)$ & $(3)$ & $(4)$ \\
\hline \multirow[t]{2}{*}{ Prediction for Female Workers } & 32.36 & 31.93 & 0.44 & 0.53 \\
\hline & $(7.71)$ & $(9.08)$ & & \\
\hline \multirow[t]{2}{*}{ Prediction for Male Workers } & 34.22 & 33.86 & 0.36 & 0.60 \\
\hline & $(7.37)$ & $(9.08)$ & & \\
\hline \multirow[t]{2}{*}{ Prediction for Indian Workers } & 35.29 & 34.31 & 0.98 & 0.21 \\
\hline & $(8.49)$ & $(10.30)$ & & \\
\hline \multirow[t]{2}{*}{ Prediction for US Workers } & 32.28 & 31.87 & 0.41 & 0.56 \\
\hline & $(8.21)$ & $(8.90)$ & & \\
\hline \multirow[t]{2}{*}{ Prediction for Over 33 Workers } & 31.95 & 31.19 & 0.75 & 0.32 \\
\hline & $(8.39)$ & $(9.58)$ & & \\
\hline \multirow[t]{2}{*}{ Prediction for Under 33 Workers } & 33.73 & 33.09 & 0.64 & 0.39 \\
\hline & $(8.58)$ & $(9.35)$ & & \\
\hline \# Observations & 290 & 287 & & \\
\hline
\end{tabular}

Notes: Standard deviations in parentheses. One observation per employer. The joint f-statistic from regression of an indicator for the "Incentivized" treatment on set of employer observable characteristics in Table B1, Panel B (duration, education, age, female, from India) is 1.25 ( $\mathrm{p}=0.286)$. 
Table C3. Discrimination in Wages, by Employee Characteristics (Hiring Task 1)

\begin{tabular}{lcccccc}
\hline & $(1)$ & $(2)$ & $(3)$ & $(4)$ & $(5)$ & $(6)$ \\
\hline Female & $-1.05^{* * *}$ & & & & $-0.67^{* * *}$ & $-0.80^{* * *}$ \\
& $(0.25)$ & & & & $(0.24)$ & $(0.19)$ \\
Indian & & $2.14^{* * *}$ & & & $1.98^{* * *}$ & $2.00^{* * *}$ \\
& & $(0.29)$ & & & $(0.29)$ & $(0.25)$ \\
Over 33 & & & $-0.54^{* *}$ & & 0.07 & 0.32 \\
& & & $(0.26)$ & & $(0.26)$ & $(0.22)$ \\
Placebo: Prefers Tea & & & & $0.52^{* *}$ & $0.39^{*}$ & $0.37^{* *}$ \\
& & & & $(0.24)$ & $(0.24)$ & $(0.18)$ \\
\hline \# Observations & 11,540 & 11,540 & 11,540 & 11,540 & 11,540 & 11,540 \\
$R^{2}$ & 0.00 & 0.01 & 0.00 & 0.00 & 0.01 & 0.49 \\
DepVarMean & 31.90 & 30.71 & 31.67 & 31.22 & 30.18 & 30.18 \\
Employer FE? & No & No & No & No & No & Yes \\
\hline${ }^{*} p<0.10,{ }^{* *} p<0.05,{ }^{* * *} p<0.01$ & & & & & &
\end{tabular}

Notes: Standard errors in parentheses, clustered by employer. "DepVarMean" is the mean of the dependent variable (wage WTP) in the omitted group (e.g. Male Workers for column (1)).

Table C4. In-Group Bias Test (Hiring Task 1)

\begin{tabular}{|c|c|c|c|c|}
\hline & (1) & $(2)$ & $(3)$ & (4) \\
\hline \multirow[t]{2}{*}{ Female Worker } & $-1.42^{* * *}$ & & & $-1.20^{* * *}$ \\
\hline & $(0.27)$ & & & $(0.27)$ \\
\hline \multirow[t]{2}{*}{ Female Employer } & $1.78^{* * *}$ & & & $1.91^{* * *}$ \\
\hline & $(0.69)$ & & & $(0.72)$ \\
\hline \multirow[t]{2}{*}{ Female Worker*Employer } & 0.26 & & & 0.41 \\
\hline & $(0.43)$ & & & $(0.42)$ \\
\hline \multirow[t]{2}{*}{ Indian Worker } & & $2.04^{* * *}$ & & $1.88^{* * *}$ \\
\hline & & $(0.31)$ & & $(0.31)$ \\
\hline \multirow[t]{2}{*}{ Indian Employer } & & 0.99 & & $1.70^{* *}$ \\
\hline & & $(0.70)$ & & $(0.74)$ \\
\hline \multirow[t]{2}{*}{ Indian Worker*Employer } & & $-0.79^{*}$ & & $-0.82^{*}$ \\
\hline & & $(0.48)$ & & $(0.48)$ \\
\hline \multirow[t]{2}{*}{ Over 33 Worker } & & & $-0.86^{* * *}$ & -0.39 \\
\hline & & & $(0.29)$ & $(0.28)$ \\
\hline \multirow[t]{2}{*}{ Over 33 Employer } & & & 0.31 & 0.22 \\
\hline & & & $(0.69)$ & $(0.71)$ \\
\hline \multirow[t]{2}{*}{ Over 33 Worker*Employer } & & & $1.10^{* * *}$ & $1.19^{* * *}$ \\
\hline & & & $(0.42)$ & $(0.41)$ \\
\hline \# Observations & 17,310 & 17,310 & 17,310 & 17,310 \\
\hline$R^{2}$ & $53^{0.01}$ & 0.01 & 0.00 & 0.02 \\
\hline DepVarMean & 31.90 & 30.71 & 31.67 & 31.67 \\
\hline
\end{tabular}

Notes: Standard errors in parentheses, clustered by employer. "DepVarMean" is the mean of the dependent variable (wage WTP) in the omitted group (e.g. Male Workers evaluated by Male Employers for column (1)). 
Table C5. In-Group vs. Out-Group Beliefs about Productivity by Employee Characteristics

\begin{tabular}{|c|c|c|c|c|c|c|}
\hline & $\begin{array}{c}\text { Out } \\
\text { Group } \\
(1)\end{array}$ & $\begin{array}{c}\text { In } \\
\text { Group } \\
(2)\end{array}$ & $\begin{array}{c}\text { Diff. } \\
(3)\end{array}$ & $\begin{array}{c}\text { p-val } \\
(4)\end{array}$ & $\begin{array}{c}\# \text { Obs. } \\
\text { Out } \\
(5)\end{array}$ & $\begin{array}{c}\# \text { Obs } \\
\text { In } \\
(6)\end{array}$ \\
\hline Prediction for Female Workers & $\begin{array}{l}31.70 \\
(8.78)\end{array}$ & $\begin{array}{l}32.79 \\
(7.81)\end{array}$ & -1.09 & 0.13 & 344 & 233 \\
\hline Prediction for Male Workers & $\begin{array}{l}34.68 \\
(6.59)\end{array}$ & $\begin{array}{l}33.60 \\
(9.20)\end{array}$ & 1.09 & 0.12 & 233 & 344 \\
\hline Prediction for Indian Workers & $\begin{array}{l}36.09 \\
(7.10)\end{array}$ & $\begin{array}{c}32.06 \\
(12.67)\end{array}$ & 4.04 & 0.00 & 392 & 185 \\
\hline Prediction for US Workers & $\begin{array}{c}30.46 \\
(12.04)\end{array}$ & $\begin{array}{l}32.84 \\
(6.15)\end{array}$ & -2.38 & 0.00 & 185 & 392 \\
\hline Prediction for Over 33 Workers & $\begin{array}{l}30.92 \\
(9.82)\end{array}$ & $\begin{array}{l}32.47 \\
(7.66)\end{array}$ & -1.55 & 0.04 & 334 & 243 \\
\hline Prediction for Under 33 Workers & $\begin{array}{l}33.85 \\
(7.03)\end{array}$ & $\begin{array}{c}33.09 \\
(10.14)\end{array}$ & 0.77 & 0.31 & 243 & 334 \\
\hline
\end{tabular}

Notes: Standard deviations in parentheses. "In-Group" refers to a match in the characteristic between the employer and the group of workers over which they are making a prediction, e.g. column (1), row 1 is the average prediction made by female employers about the average productivity of female workers. 